\title{
Accuracy of one-step integration schemes for damped/forced linear structural dynamics
}

\author{
Alexandre Depouhon ${ }^{1,2}$, Emmanuel Detournay ${ }^{2}$ and Vincent Denoël ${ }^{1},{ }^{*}$ \\ ${ }^{1}$ Structural Engineering, ArGEnCo, Department of Applied Sciences, Université de Liège, Belgium \\ ${ }^{2}$ Department of Civil Engineering, University of Minnesota, MN, USA
}

\begin{abstract}
SUMMARY
This paper proposes an energy-based measure for the evaluation of the local truncation error of two-level one-step integration schemes. The measure is applicable to multiple degree of freedom systems and, as such, does not necessarily require the reduction of the problem to the dynamics of a single mode by the invocation of orthogonality arguments; for a consistent treatment of the problem, it naturally handles the structural damping and external forcing terms which are generally and mistakenly neglected in error analyses; and it segregates the error associated with the free and forced response components of the problem. To illustrate the approach, two examples associated with the application of the trapezoidal scheme and of a high-order scheme proposed in the literature are analyzed. The latter example reveals the shortcomings of the standard approach that is based on the undamped/unforced linear oscillator and therefore highlights the need for the proposed framework. In this example, the scheme order of accuracy is, indeed, below expectation when applied to an oscillator subject to structural damping or external forcing, in the numerically dissipative setting.

A reformulation of the deficient scheme, enabling the recovery of its high-order accuracy, is proposed. It is obtained by demonstrating its equivalence to a four-level one-step scheme related to the time discontinuous Galerkin (TDG) method. Steps for providing the corrective terms include $(i)$ the presentation and analysis of three four-level schemes related to the TDG method for application in linear structural dynamics and (ii) the recasting in two-level form of one of these schemes by elimination of the internal variables via static condensation.
\end{abstract}

Copyright (c) 0000 John Wiley \& Sons, Ltd.

Received ...

KEY WORDS: one-step time integration schemes; structural dynamics; order of accuracy; external forcing; damping; local truncation error

*Correspondence to: Vincent Denoël, Structural Engineering, ArGEnCo, Department of Applied Sciences, Université de Liège, B52, 1 Chemin des Chevreuils, 4000 Liège, Belgium. E-mail: v.denoel@ulg.ac.be

Copyright (C) 0000 John Wiley \& Sons, Ltd.

Prepared using nmeauth.cls [Version: 2010/05/13 v3.00] 


\section{INTRODUCTION}

As per Hilber and Hughes [1], competitive time integration schemes devoted to structural dynamics should verify the following list of attributes:

1. Unconditional stability when applied to linear problems.

2. No more than one set of implicit equations should have to be solved at each step.

3. Second-order accuracy, at least.

4. Controllable algorithmic dissipation in the higher modes.

5. Self-starting.

On the one hand, some of these points can be fulfilled a priori by choices at the development or selection stage of the time integration procedure, notably of the principle underlying the discretization of the equations of motion. For instance, item 1. precludes explicit algorithms that are only conditionally stable [2]; item 5 . rules out the use of linear multistep solvers for time integration in structural dynamics; the use of the generalized framework for one-step three-level schemes (the number of levels is defined as the ratio between the state-vector dimension and the number of degrees of freedom in the problem [3]) proposed by Zhou and Tamma [4] enables the translation of these criteria into constraints on the generalized scheme parameter sets and, thus, their verification at the design level. On the other hand, the stability and accuracy properties of integration schemes, items 1. and 3., are typically evaluated at a subsequent stage of the development of an integration procedure. The importance of their correct assessment stems from two purposes: $(i)$ the establishment of the procedure convergence that is guaranteed by its consistency and stability (Lax-Richtmyer equivalence theorem [5]), and (ii) the selection criteria for choosing an integration scheme for a specific application partly rely on these properties.

The commonly adopted criterion for stability is that the numerical solution should remain uniformly bounded over all computed time points [3]. Although not fully equivalent in the nonlinear context [6], this measure is usually superseded by the natural notion of algorithmic stability related to the conservation or decay of the system mechanical energy along the computed system trajectory [7]. Verification of the non-increasing character of the system mechanical energy (and possibly that of other relevant physical quantities such as momentum) has served as a design basis for problem-specific algorithms, e.g., the secondorder accurate energy-dissipative momentum-conserving (EDMC-2) scheme proposed by Armero and Romero [8] for nonlinear elastodynamics or the unconditionally stable scheme of Bottasso and Borri [9] dedicated to the handling of flexible beams undergoing finite placement. Similar energy arguments can be developed in the linear context, in which energy boundedness does guarantee that of the solution. They are considerably simpler though; see, for instance, the energy-based stability analysis of Newmark-related integration schemes in [10]. Further simplification even follows if, in addition, the equations of motion can be uncoupled through modal expansion $[11,12]$. The problem can then be essentially reduced to a single degree of freedom oscillator and the stability of the integration scheme assessed from the spectral properties of the associated amplification matrix $[3,13,14,15]$; the integration scheme is stable provided the amplification matrix has eigenvalues within the unit circle, with at most one eigenvalue with unit modulus. 
In view of the simplicity of the spectral analysis on the single degree of freedom model, relative to energy-based arguments, the scalar oscillator, representative of a single mode dynamics, has become, de facto, the model linear problem for the assessment of a scheme accuracy. Moreover, as the stability analysis is concerned with internal stability and as the undamped oscillator is often mistakenly assumed to be the worst-case scenario in regards to stability bounds [16], structural damping and external forcing are usually not accounted for in assessing the order of accuracy of an integration scheme; see for instance these analyses of one-step schemes that rest on the scalar undamped/unforced oscillator $[8,13,17,18,19]$.

To ensure the consistency of the analysis with respect to the operational usage of the integration procedure, the local truncation error should, however, be evaluated on the basis of the damped/forced problem, as recommended by Wood [20] who provides a framework for the analysis of displacement-based multistep schemes. While some authors do account for structural damping in their local truncation error analysis [12, 15, 21, 22], very few do so for the external forcing, as noted by Fung [23]. This might provide a wrong picture of the LTE of these algorithms.

To streamline the consistent assessment of the order of accuracy of one-step integration schemes for linear dynamics - this paper does not deal with overshooting issues that commonly arise with schemes that preserve an algorithmic quantity rather than the mechanical energy, e.g., Newmark-based schemes [10]—we present a novel framework for the evaluation of the local truncation error of two-level schemes. The proposed framework relies on the evaluation of two error norms relative to the free (homogeneous) and forced (particular) response components. These error norms are proportional to the local truncation error and provide an efficient way of evaluating the scheme order of accuracy with respect to the free and forced response components. The actual scheme order of accuracy, in operating conditions, then appears to be the minimum of the two values. Also, the influence of structural damping is naturally accounted for in the error analysis, making it a complete assessment of the scheme accuracy. Contrary to Wood's framework [20], ours is directly applicable to two-level one-step schemes; it does not require their recasting into an equivalent displacement-based multistep form, which makes the analysis simpler, not only analytically but also numerically.

To illustrate the analysis appoach, two examples are considered. First, the method recovers the second-order accuracy of the trapezoidal scheme when applied to the linear damped and forced oscillator using an analytical application of the framework; constant and sinusoidal loadings are considered. Second, we demonstrate the possible shortcomings of the traditional approach that considers the undamped/unforced problem as reference by the numerical application of our framework to the high-order scheme proposed by Krenk [19]. The thorough analysis conducted by the author on the undamped/unforced linear oscillator predicts that the scheme achieves fourth-order and third-order accurate integration in the numerically conservative and dissipative settings, respectively. These results are confirmed for that specific oscillator configuration. However, they are invalidated when the scheme is used in its numerically dissipative setting, in the presence of structural damping or external forcing. 
With the aim of correcting the formulation of the latter high-order scheme, the paper proposes two additional contributions. First, we introduce the linear time discontinuous Galerkin (TDG) method and two four-level one-step schemes that relate to the TDG method when applied to linear problems, although they were developed independently for specific nonlinear problems, see [8, 24, 25]. Our presentation extends that of Bottasso and Trainelli [26] to the case of damped and forced linear structural systems. Second, we prove the equivalence between one of the TDG-like schemes and the one proposed by Krenk [19], in the undamped/unforced case. Then, extending the equivalence to other configurations of the model oscillator, the corrections necessary for the recovery of its high-order accuracy are identified.

The paper is organized as follows. Section 2 introduces the framework for the accuracy analysis and develops the two example applications. Presentation of the TDG-like schemes comes in Section 3, followed by the establishment of the equivalence with Krenk's scheme and the identification of the corrective terms required for the recovery of the scheme accuracy in the numerically dissipative setting, in the presence of structural damping or external forcing, in Section 4. The paper then closes with a summary of the results.

\section{CALCULATION OF THE LOCAL TRUNCATION ERROR}

Let us consider the model problem of linear structural dynamics under state-space form

$$
\left(\begin{array}{c}
\dot{\mathbf{u}} \\
\dot{\mathbf{v}}
\end{array}\right)=\left(\begin{array}{cc}
\mathbf{0} & \mathbf{I} \\
-\mathbf{M}^{-1} \mathbf{K} & -\mathbf{M}^{-1} \mathbf{C}
\end{array}\right)\left(\begin{array}{l}
\mathbf{u} \\
\mathbf{v}
\end{array}\right)+\left(\begin{array}{l}
\mathbf{0} \\
\mathbf{a}
\end{array}\right),
$$

or

$$
\dot{\mathbf{x}}=\mathbf{F} \mathbf{x}+\mathbf{g}
$$

in shorthand notation. Vectors $\mathbf{u}(t), \mathbf{v}(t), \mathbf{a}(t):=\mathbf{M}^{-1} \mathbf{f}(t)$ refer to the displacement and velocity fields, and the external acceleration resulting from the forcing $\mathbf{f}(t)$, respectively; column vector $\mathbf{x}=[\mathbf{u} ; \mathbf{v}]$ denotes the state vector. The overhead dot denotes differentiation with respect to time $\dot{x}=\mathrm{d} x / \mathrm{d} t$. The mass matrix is assumed symmetric positive definite, $\mathbf{M}=\mathbf{M}^{T}, \mathbf{M}>0$, while the damping and stiffness matrices are assumed symmetric positive semi-definite, $\mathbf{Z}=\mathbf{Z}^{T}, \mathbf{Z} \geq 0, \mathbf{Z} \in\{\mathbf{C}, \mathbf{K}\}$. This guarantees the negative semi-definiteness of matrix $\mathbf{F}$ and the system internal stability, i.e., the system state remains bounded as time goes to infinity. Such assumptions are usually verified when the governing matrices arise from a space semi-discretization using the finite element method.

From the theory of linear time-invariant (LTI) systems, the analytical solution to (2), $\underline{\mathbf{x}}(t)$, is known as [27]

$$
\underline{\mathbf{x}}(t)=\mathrm{e}^{\mathbf{F}\left(t-t_{0}\right)} \mathbf{x}\left(t_{0}\right)+\int_{t_{0}}^{t} \mathrm{e}^{\mathbf{F}(t-\tau)} \mathbf{g}(\tau) \mathrm{d} \tau
$$

Copyright (c) 0000 John Wiley \& Sons, Ltd.

Prepared using nmeauth.cls
Int. J. Numer. Meth. Engng (0000)

DOI: $10.1002 / \mathrm{nme}$ 
It is the sum of the homogeneous and particular responses of the dynamical system, respectively related to the state-transition matrix $\mathrm{e}^{\mathbf{F}\left(t-t_{0}\right)}$ and to the continuous-time convolution (integral) product between the system impulse response and the input vector $\mathrm{g}:=[\mathbf{0} ; \mathbf{a}]$.

An alternative solution relies on discretizing the underlying governing problem via, among others, finite elements $[17,22]$ or finite differences $[28,29]$ and approximating the continuous integration by a step-by-step update scheme. The evolution equation resulting from the application of one-step schemes to equation (2) can be cast in the generic form

$$
\mathbf{H}_{0} \mathbf{x}_{n+1}=\mathbf{H}_{1} \mathbf{x}_{n}+\ell_{n}^{n+1}
$$

where $\mathbf{H}_{0}, \mathbf{H}_{1}$ are the iteration matrices and $\boldsymbol{\ell}_{n}^{n+1}$ is the load vector that accounts for the external loading over the time interval $t \in\left[t_{n}, t_{n+1}\right]$. Additionally to the displacement and velocity variables, the state vector $\mathbf{x}_{n}:=\mathbf{x}\left(t_{n}\right)$ may contain higher-order field variables, e.g., the acceleration in schemes of the $\alpha$-family $[15,30,31]$, or internal variables in schemes related to the time discontinuous Galerkin method [17, 32]. The ratio between the dimension of the state vector and the number of degrees of freedom of the system defines the number of levels $m$ of the scheme. The iteration matrices and the load vector are entirely defined by the integration scheme. They generally depend on the governing matrices $(\mathbf{M}, \mathbf{C}, \mathbf{K})$, the timestep $(h)$, the external nodal loads (a) and, possibly, on algorithmic parameters related to the scheme.

Upon solving for the state vector at the end of the timestep, $\mathbf{x}_{n+1}$, the update equation (4) takes the explicit form

$$
\mathbf{x}_{n+1}=\mathbf{A} \mathbf{x}_{n}+\mathbf{b}_{n}^{n+1},
$$

with amplification matrix $\mathbf{A}:=\mathbf{H}_{0}^{-1} \mathbf{H}_{1}$ and direct load vector $\mathbf{b}_{n}^{n+1}:=\mathbf{H}_{\mathbf{0}}{ }^{-1} \ell_{n}^{n+1}$. It is the spectral and asymptotic characteristics of these update components that define the scheme stability conditions and order of accuracy [14].

\subsection{Spectral analysis}

The conventional steps of a spectral analysis are:

(i) The reduction of the multiple degree of freedom problem (1) to a single degree of freedom problem by invoking eigenmode orthogonality whenever the damping matrix shares the eigenvectors of the mass and stiffness matrices [11, 12]. The governing matrices become scalar variables. The problem is usually normalized to have unit mass

$$
\mathbf{M} \leftarrow 1, \quad \mathbf{C} \leftarrow 2 \zeta \omega_{0}, \quad \mathbf{K} \leftarrow \omega_{0}^{2}, \quad \mathbf{a} \leftarrow a .
$$

(ii) The single degree of freedom model is considered in its undamped/unforced version

$$
\zeta \leftarrow 0, \quad a \leftarrow 0 .
$$

(iii) The one-step scheme is applied to the model problem to form the $m \times m$ amplification matrix. 
(iv) The eigenvalues of the amplification matrix are computed. Stability conditions are established on the basis of their location in the complex plane.

$(v)$ The scheme order of accuracy with respect to the timestep $h$ is calculated from its local truncation error (LTE). The numerical damping and the relative period elongation (numerical dispersion) introduced by the scheme are reported as a function of the reduced eigenfrequency $\Omega_{0}:=\omega_{0} h$. Convergence of the scheme is then ensured provided it is stable and has a strictly positive order of accuracy, in virtue of the Lax-Richtmyer theorem [5].

Additional details and considerations about steps $(i v),(v)$ can be found in the books $[5,12$, $14,33]$ and papers $[13,19,21,22]$, among others texts.

However, as was already noted by Wood [34], the presence of natural damping in the equation of motion of the oscillator, may influence the order of accuracy and the stability conditions of an integration scheme. Furthermore, it is of general interest to engineers to compute the response of structures to external dynamic loads. As such, accuracy properties of integration schemes should be evaluated in the presence of a forcing term as well.

To that end, Wood [20] proposes a framework for the analysis of linear multistep methods as applied to the damped scalar oscillator under harmonic forcing; that is, above step $(i i)$ is replaced by $\zeta \leftarrow \zeta_{*}>0, f \leftarrow \mathrm{e}^{\mathrm{i} p t}, \mathrm{i}=\sqrt{-1}$. As the framework is directed towards the analysis of displacement-based multistep schemes, it is not directly applicable to one-step schemes that rely on evolution equations involving displacement and velocity variables. Reduction of multi-level one-step schemes to their equivalent multistep form is thus required prior to the application of Wood's framework, a manipulation that can prove cumbersome, especially for high-order schemes involving internal variables. This and the lack, to the authors' knowledge, of a proper framework for the analysis of the local truncation error in one-step integration schemes prompt for the method we propose in the next section.

\subsection{Proposed framework for the local truncation error analysis}

There is no possible discussion about the analysis of the internal stability of equation (5). Its homogeneous response is given by

$$
\mathbf{x}_{n}=\mathbf{A}^{n} \mathbf{x}_{0}
$$

and remains bounded provided the spectral radius of the amplification matrix is bounded by 1 and that eigenvalues with unit modulus have unit multiplicity at most. However, when it comes to the evaluation of the scheme accuracy, analyses based on the sole eigenvalues of the amplification matrix are debatable. They indeed discard spurious eigenvalues and assume the motion or state vector to be parallel to the eigenvector of the principal eigenvalue of the amplification matrix, an assumption that is not always met in practice; see for instance [35], where this issue is addressed for the generalized- $\alpha$ method. Also, if the analysis neglects the

forced response of the system, inconsistencies in the formation of load vector $\ell_{n}^{n+1}$ can go unnoticed and the scheme could suffer a loss of accuracy due to the improper handling of forcing terms; this issue is examined later on. 
Let us now define the energy-based error measure

$$
e(t):=\frac{\sqrt{2}}{2}\left\|\boldsymbol{\Gamma}^{1 / 2} \mathbf{e}_{x}(t)\right\|_{2},
$$

with the symmetric positive semi-definite (in virtue of the assumptions on the mass and stiffness matrices) block diagonal scaling matrix $\boldsymbol{\Gamma}:=\operatorname{diag}(\mathbf{K}, \mathbf{M})$ and the error vector on the displacement and velocity fields $\mathbf{e}_{x}(t)=[\mathbf{u}(t)-\underline{\mathbf{u}}(t) ; \mathbf{v}(t)-\underline{\mathbf{v}}(t)]$; the underlined variables refer to the analytical solution (3), the regular notation to the numerical solution. This norm has been introduced by Romero [36] as the natural measure to quantify the errors arising from the numerical computation of the motion of mechanical systems. It can be interpreted as the mechanical energy associated with the errors on the displacement and velocity fields.

Considering the error generated by a single increment of the integration procedure from initial time 0 (without loss of generality), i.e., $\mathbf{x}_{0}=\underline{\mathbf{x}}_{0}=\underline{\mathbf{x}}(0)$, the error measure can be specialized to

$$
\begin{aligned}
e(h) & =\frac{\sqrt{2}}{2}\left\|\boldsymbol{\Gamma}^{1 / 2}\left(\left(\mathbf{A}-\mathrm{e}^{\mathbf{F} h}\right) \underline{\mathbf{x}}_{0}+\mathbf{b}_{0}^{1}-\int_{0}^{h} \mathrm{e}^{\mathbf{F}(h-\tau)} \mathbf{g}(\tau) \mathrm{d} \tau\right)\right\|_{2} \\
& \leq \frac{\sqrt{2}}{2}\left\|\boldsymbol{\Gamma}^{1 / 2}\left(\mathbf{A}-\mathrm{e}^{\mathbf{F} h}\right) \underline{\mathbf{x}}_{0}\right\|_{2}+\frac{\sqrt{2}}{2}\left\|\boldsymbol{\Gamma}^{1 / 2}\left(\mathbf{b}_{0}^{1}-\int_{0}^{h} \mathrm{e}^{\mathbf{F}(h-\tau)} \mathbf{g}(\tau) \mathrm{d} \tau\right)\right\|_{2}
\end{aligned}
$$

by use of the expressions for the numerical and analytical solutions, and of the triangle inequality.

Thus, the total numerical error arising from a single timestep can be decomposed into two components, relative to the free and forced responses of the system. The first component is evidently proportional to the initial energy of the system $E_{0}:=\left\|\boldsymbol{\Gamma}^{1 / 2} \mathbf{x}_{0}\right\|^{2} / 2$. Further hypothesizing the positive definiteness of the scaling matrix $\boldsymbol{\Gamma}$, a condition that follows from the assumption of positive definiteness of the stiffness matrix $\mathbf{K}>0$, we free ourselves from this dependency by maximizing it over all initial conditions with unit energy $E_{0}=1$. This yields

$$
e_{1}(h):=\left\|\boldsymbol{\Gamma}^{1 / 2}\left(\mathbf{A}-\mathrm{e}^{\mathbf{F} h}\right) \boldsymbol{\Gamma}^{-1 / 2}\right\|_{2},
$$

by definition of the matrix 2-norm (maximum singular value) [5, Theorem 1.2]. As to the second component, it is defined as

$$
e_{2}(h):=\frac{\sqrt{2}}{2}\left\|\boldsymbol{\Gamma}^{1 / 2}\left(\mathbf{b}_{0}^{1}-\int_{0}^{h} \mathrm{e}^{\mathbf{F}(h-\tau)} \mathbf{g}(\tau) \mathrm{d} \tau\right)\right\|_{2}
$$

and is problem specific through the definition of the external loading $\mathbf{g}(t)$.

Error components $e_{1}(h), e_{2}(h)$ are easily identified as being proportional to the local truncation error as defined by Hughes [14] and to the timestep $h$. The leading-order terms of their Taylor expansion around $h=0$ therefore directly yields the scheme orders of accuracy $k_{1}, k_{2}$ as regards the free and forced computed responses

$$
e_{1}(h) \sim C_{1} h^{k_{1}+1}, \quad e_{2}(h) \sim C_{2} h^{k_{2}+1} .
$$


Given the additive nature of the errors, the overall order of accuracy of the scheme $k$ is given by

$$
k:=\min \left\{k_{1}, k_{2}\right\}
$$

Constants $C_{1}, C_{2}$ depend on the model parameters and, possibly, on the algorithmic parameters related to the integration scheme. They merely follow from the limiting behavior of $e_{1}(h), e_{2}(h)$ when the timestep tends to 0

$$
C_{1}=\lim _{h \rightarrow 0} \frac{e_{1}(h)}{h^{k_{1}+1}}, \quad C_{2}=\lim _{h \rightarrow 0} \frac{e_{2}(h)}{h^{k_{2}+1}} .
$$

Under the assumptions of well-definiteness of the governing matrices, the above developments do apply to linear structural dynamics as a whole, not only to models for which modal expansion applies. Tractability matters, nonetheless, encourage the use of a single degree of freedom model whenever possible. In that context, analytical developments remain accessible. In particular, the treatment of loadings of polynomial or periodic nature can be conducted analytically; it is developed in the next section. However, parallel to the analytical route, the numerical one remains the fastest and most versatile one, as all configurations can be treated; the convolution product will then be computed using adaptive quadrature with stringent tolerances to reach error levels close to machine epsilon. A linear regression of the error components versus the timestep, after logarithmic transformation, provides approximations to $k_{1}, k_{2}, C_{1}, C_{2}$.

\subsection{Matrix exponential and convolution products for the analytical calculation of $e_{1}, e_{2}$}

The evaluation of the error norms $e_{1}(h), e_{2}(h)$ involves that of the state-transition matrix $\mathrm{e}^{\mathbf{F} t}$ of LTI system (2) and the calculation of the convolution product associated with the forced response of the system. While the expression of the state-transition matrix merely follows from its definition, the calculation of the convolution product can prove an arduous task for loadings having complex time evolutions. Nevertheless, it remains tractable for two main classes of time evolutions: (i) polynomial loadings (or loadings that can be expanded in power series) and ( $i i)$ periodic loadings. These developments are now proposed on the basis of the scalar model

$$
\left(\begin{array}{l}
\dot{u} \\
\dot{v}
\end{array}\right)=\left(\begin{array}{cc}
0 & 1 \\
-\omega_{0}^{2} & -2 \zeta \omega_{0}
\end{array}\right)\left(\begin{array}{l}
u \\
v
\end{array}\right)+\left(\begin{array}{l}
0 \\
a
\end{array}\right) .
$$

\section{The matrix exponential}

The state-transition matrix (matrix exponential) is easily computed by spectral decomposition, yielding

$$
\mathrm{e}^{\mathbf{F} t}=\mathrm{e}^{-\zeta \omega_{0} t}\left(\begin{array}{cc}
\cos \omega_{d} t+\frac{\zeta}{\sqrt{1-\zeta^{2}}} \sin \omega_{d} t & \frac{1}{\omega_{d}} \sin \omega_{d} t \\
-\frac{\omega_{0}}{\sqrt{1-\zeta^{2}}} \sin \omega_{d} t & \cos \omega_{d} t-\frac{\zeta}{\sqrt{1-\zeta^{2}}} \sin \omega_{d} t
\end{array}\right),
$$


with $\omega_{d}:=\omega_{0} \sqrt{1-\zeta^{2}}$ (subcritical damping is assumed, i.e., $\zeta \in[0,1]$ ). This expression enters the definition of both $e_{1}(h), e_{2}(h)$.

\section{The convolution product}

The model problem being LTI, the superposition principle applies to the calculation of the forced response. Accordingly, if the time variation of the loading term can be expressed as a partial sum of basis functions $\varphi_{n}(t)$,

$$
a(t)=\sum_{n \in \mathcal{N}} \mu_{n} \varphi_{n}(t)
$$

the linearity of the integration operator underlying the convolution product can be exploited to calculate it, i.e.,

$$
a(t) * g(t)=\sum_{n \in \mathcal{N}} \mu_{n}\left(\varphi_{n}(t) * g(t)\right),
$$

where the star operator denotes continuous-time convolution.

Also, given the trigonometric nature of the state-transition matrix entries, the specific form of the loading vector $\mathbf{g}(t)=[0 ; a(t)]$, and recalling that sine and cosine functions can be expressed as linear combinations of complex exponentials, namely

$$
\sin \omega_{d} t=\frac{1}{2 \mathrm{i}}\left(\mathrm{e}^{\mathrm{i} \omega_{d} t}-\mathrm{e}^{-\mathrm{i} \omega_{d} t}\right), \quad \cos \omega_{d} t=\frac{1}{2}\left(\mathrm{e}^{\mathrm{i} \omega_{d} t}+\mathrm{e}^{-\mathrm{i} \omega_{d} t}\right),
$$

the calculation of the convolution product essentially boils down to finding an analytical expression for

$$
H^{ \pm}\left[\varphi_{n}(t)\right]=\int_{0}^{h} \mathrm{e}^{\left(-\zeta \omega_{0} \pm \mathrm{i} \omega_{d}\right)(h-\tau)} \varphi_{n}(\tau) \mathrm{d} \tau
$$

More specifically, if we denote by $(\mathbf{A})_{i j}$ the $i^{\text {th }}$-row $j^{\text {th }}$-column entry of matrix $\mathbf{A}$, the two entries of the convolution product read

$$
\begin{aligned}
\int_{0}^{h}\left(\mathrm{e}^{\mathbf{F}(h-\tau)}\right)_{12} \varphi_{n}(\tau) \mathrm{d} \tau & =\frac{1}{2 \mathrm{i} \omega_{d}}\left(H^{+}\left[\varphi_{n}(t)\right]-H^{-}\left[\varphi_{n}(t)\right]\right) \\
\int_{0}^{h}\left(\mathrm{e}^{\mathbf{F}(h-\tau)}\right)_{22} \varphi_{n}(\tau) \mathrm{d} \tau & =\frac{-\zeta+\mathrm{i} \sqrt{1-\zeta^{2}}}{2 \mathrm{i} \sqrt{1-\zeta^{2}}} H^{+}\left[\varphi_{n}(t)\right]+\frac{\zeta+\mathrm{i} \sqrt{1-\zeta^{2}}}{2 \mathrm{i} \sqrt{1-\zeta^{2}}} H^{-}\left[\varphi_{n}(t)\right]
\end{aligned}
$$

Assuming the external load to be real, entries $H^{ \pm}\left[\varphi_{n}(t)\right]$ are complex conjugate and the above expressions further simplify into

$$
\begin{aligned}
\int_{0}^{h}\left(\mathrm{e}^{\mathbf{F}(h-\tau)}\right)_{12} \varphi_{n}(\tau) \mathrm{d} \tau & =\frac{1}{\omega_{d}} H_{\operatorname{Im}}^{+}\left[\varphi_{n}\right] \\
\int_{0}^{h}\left(\mathrm{e}^{\mathbf{F}(h-\tau)}\right)_{22} \varphi_{n}(\tau) \mathrm{d} \tau & =H_{\operatorname{Re}}^{+}\left[\varphi_{n}\right]-\frac{\zeta}{\sqrt{1-\zeta^{2}}} H_{\operatorname{Im}}^{+}\left[\varphi_{n}\right]
\end{aligned}
$$

where $H_{\mathrm{Re}}^{+}\left[\varphi_{n}(t)\right], H_{\mathrm{Im}}^{+}\left[\varphi_{n}(t)\right]$ denote the real and imaginary parts of $H^{+}\left[\varphi_{n}(t)\right]$, respectively. 
In the case of polynomial loadings or smooth functions that can be expanded in power series $(N=+\infty)$, the basis functions are the powers of the time variable, $\varphi_{n}(t)=t^{n}$, and

$$
a(t)=\sum_{n=0}^{N} \mu_{n} t^{n} .
$$

Defining the incomplete gamma function

$$
\gamma(n+1, x)=\int_{0}^{x} t^{n} \mathrm{e}^{-t} \mathrm{~d} t
$$

it is straightforward to establish that

$$
H^{ \pm}\left[t^{n}\right]=\int_{0}^{h} \mathrm{e}^{\left(-\zeta \omega_{0} \pm \mathrm{i} \omega_{d}\right)(h-\tau)} \tau^{n} \mathrm{~d} \tau=\gamma\left(n+1,-\zeta \Omega_{0} \pm \mathrm{i} \Omega_{d}\right) \frac{\mathrm{e}^{-\zeta \Omega_{0} \pm \mathrm{i} \Omega_{d}}}{\left(-\zeta \omega_{0} \pm \mathrm{i} \omega_{d}\right)^{n+1}},
$$

where $\Omega_{0}:=\omega_{0} h, \Omega_{d}:=\omega_{d} h$. Substitution in equations (22)-(23) then provides the expressions of the convolution product.

In the case of a periodic loading with period $T$, the time variation of the forcing term can be expressed as a Fourier sum

$$
a(t)=\sum_{n=-N}^{N} \mu_{n} \mathrm{e}^{\mathrm{i} \omega_{n} t}
$$

with

$$
\omega_{n}=\frac{2 \pi n}{T}, \quad \mu_{n}=\frac{1}{T} \int_{-T / 2}^{T / 2} f(t) \mathrm{e}^{-\mathrm{i} \omega_{n} t} \mathrm{~d} t .
$$

Thus, $\varphi_{n}(t)=\mathrm{e}^{\mathrm{i} \omega_{n} t}$. The convolution product is then obtained from (22)-(23) using the following result

$$
H^{ \pm}\left[\mathrm{e}^{\mathrm{i} \omega t}\right]=\int_{0}^{h} \mathrm{e}^{\left(-\zeta \omega_{0} \pm \mathrm{i} \omega_{d}\right)(h-\tau)} \mathrm{e}^{\mathrm{i} \omega \tau} \mathrm{d} \tau=\frac{\mathrm{e}^{\mathrm{i} \Omega}-\mathrm{e}^{-\zeta \Omega_{0} \pm \mathrm{i} \Omega_{d}}}{\mathrm{i}\left(\omega \mp \omega_{d}\right)+\zeta \omega_{0}}
$$

with $\Omega:=\omega h$. Note that the above results are not valid when, in the absence of natural damping $(\zeta=0)$, the oscillator is excited at its resonance frequency, as this translates into the unboundedness of $H^{ \pm}\left[\mathrm{e}^{\mathrm{i} \omega t}\right]$.

\subsection{Application examples}

To illustrate the proposed framework, we evaluate the accuracy of two integration schemes for structural dynamics. Analytical developments are conducted for the well-known secondorder trapezoidal scheme. A numerical approach is followed instead in assessing the accuracy of the high-order scheme proposed by Krenk [19].

\section{Trapezoidal scheme}

The trapezoidal scheme follows from the integration of equations of motion (1) under the assumption of linear time evolution of the displacement and velocity fields. It results in the 
approximation

$$
\left(\begin{array}{cc}
\frac{h}{2} \mathbf{K} & \mathbf{M}+\frac{h}{2} \mathbf{C} \\
\mathbf{I} & -\frac{h}{2} \mathbf{I}
\end{array}\right)\left(\begin{array}{c}
\mathbf{u}_{n+1} \\
\mathbf{v}_{n+1}
\end{array}\right)=\left(\begin{array}{cc}
-\frac{h}{2} \mathbf{K} & \mathbf{M}-\frac{h}{2} \mathbf{C} \\
\mathbf{I} & \frac{h}{2} \mathbf{I}
\end{array}\right)\left(\begin{array}{c}
\mathbf{u}_{n} \\
\mathbf{v}_{n}
\end{array}\right)+\left(\begin{array}{c}
\frac{h}{2}\left(\mathbf{f}_{n}+\mathbf{f}_{n+1}\right) \\
\mathbf{0}
\end{array}\right),
$$

which is known to be second-order accurate, unconditionally stable and numerically conservative, as the eigenvalues of the amplification matrix are complex conjugate with unit magnitude for all frequencies. Reduction to the damped/forced scalar oscillator leads to the following amplification matrix and direct load vector

$$
\mathbf{A}=\frac{1}{D}\left(\begin{array}{cc}
4+4 \zeta \Omega_{0}-\Omega_{0}^{2} & 4 h \\
-4 \Omega_{0} \omega_{0} & 4-4 \zeta \Omega_{0}-\Omega_{0}^{2}
\end{array}\right), \quad \mathbf{b}_{0}^{1}=\frac{1}{D}\left(\begin{array}{c}
h^{2} \\
2 h
\end{array}\right)\left(a_{n}+a_{n+1}\right),
$$

with denominator $D=4+4 \zeta \Omega_{0}+\Omega_{0}^{2}, \Omega_{0}:=\omega_{0} h$ and state vector $\mathbf{x}_{n}=\left[u_{n} ; v_{n}\right]$.

Completing all algebra, the Maclaurin expansion of $e_{1}(h)$ reads

$$
e_{1}(h)=\frac{\omega_{0}^{3} h^{3}}{12} \sqrt{\eta+\sqrt{\eta^{2}-1}}+\mathcal{O}\left(h^{4}\right), \quad \eta(\zeta)=1+2 \zeta^{2}-16 \zeta^{4}+32 \zeta^{6} .
$$

Thus, $k_{1}=2$ and $C_{1}=\omega_{0}^{3} \sqrt{\eta+\sqrt{\eta^{2}-1}} / 12$.

The error on the forced response is dependent on the type of loading. As example results, we provide the error for constant $\left(a(t)=a_{0}\right)$ and sinusoidal $\left(a(t)=a_{0} \sin \omega t, \omega \neq \omega_{0}\right)$ loadings, assuming zero phase for simplicity. They respectively read

$$
\begin{aligned}
& e_{2}(h)=\frac{\sqrt{1-4 \zeta^{2}+16 \zeta^{4}}}{12 \sqrt{2}} a_{0} \omega_{0}^{2} h^{3}+\mathcal{O}\left(h^{4}\right), \\
& e_{2}(h)=\frac{\sqrt{1+4 \zeta^{2}}}{12 \sqrt{2}} a_{0} \omega_{0} \omega h^{3}+\mathcal{O}\left(h^{4}\right),
\end{aligned}
$$

by application of equations (18), (22)-(23), (28), (31). Accordingly, for both loading types, we have $k_{2}=2$ and the leading-order constants are identified as $C_{2}=$ $\left(a_{0} \omega_{0}^{2} \sqrt{1-4 \zeta^{2}+16 \zeta^{4}}\right) /(12 \sqrt{2})$ and $C_{2}=\left(a_{0} \omega_{0} \omega \sqrt{1+4 \zeta^{2}}\right) /(12 \sqrt{2})$, respectively.

As $k=\min \left\{k_{1}, k_{2}\right\}=2$, the trapezoidal scheme is confirmed to achieve second-order accuracy on linear problems. The order of accuracy is independent of the presence of damping (leading-order constants do not vanish when $\zeta=0$ ) or external forcing $\left(k_{1}=k_{2}\right)$, the latter case having been verified for constant and sinusoidal loads. 

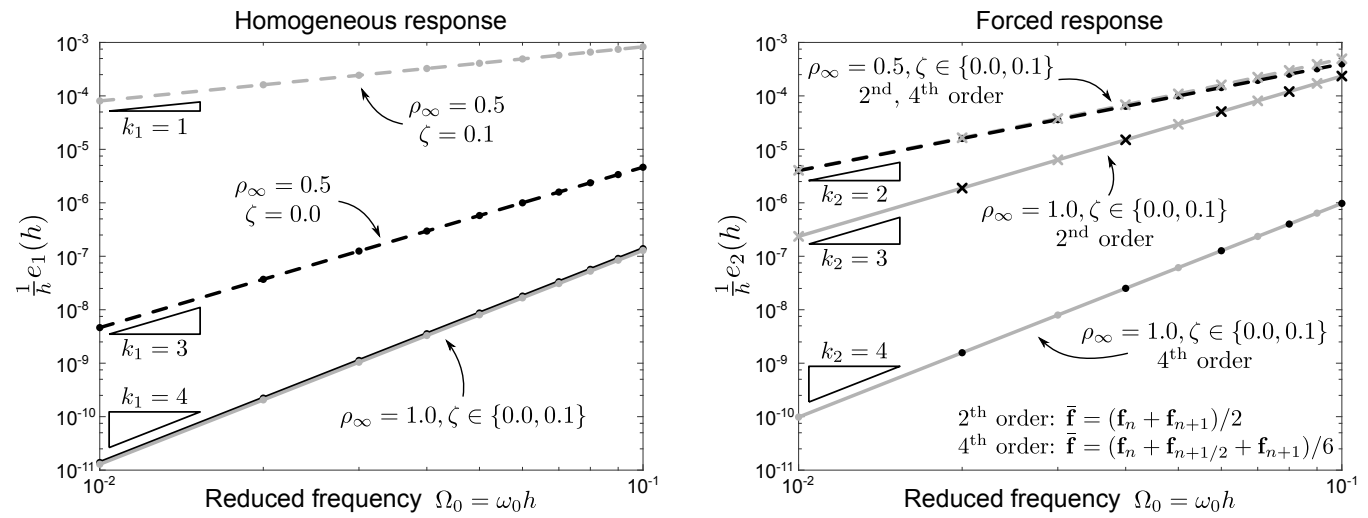

Figure 1. Scaled error dependency on the timestep using Krenk's scheme on the scalar model problem with sinusoidal excitation $(a(t)=\sin \omega t)$ and model parameters $\left(\omega_{0}, \omega\right)=(1,2)$. Line slopes represent the orders of accuracy $k_{1}, k_{2}$. The scheme is fourth-order accurate in the numerically conservative setting $\left(\rho_{\infty}=1.0\right)$ when high-order integration is used to evaluate the average forcing term; it is third-order accurate in case low-order integration is exploited. In the numerically dissipative setting $\left(\rho_{\infty}<1.0\right)$, the scheme order of accuracy drops to two and one in the absence $(\zeta=0.0)$ and presence $(\zeta=0.1>0)$ of structural damping, respectively.

Krenk's scheme [19]

On the basis of the integration by parts of equation (1), Krenk has proposed the following two-level one-step integration scheme

$$
\begin{gathered}
\left(\begin{array}{cc}
\mathbf{C}+\left(\frac{1}{2}+\chi\right) h \mathbf{K} & \mathbf{M}-\left(\frac{1}{12}+\frac{1}{2} \chi\right) h^{2} \mathbf{K} \\
\mathbf{M}-\left(\frac{1}{12}+\frac{1}{2} \chi\right) h^{2} \mathbf{K} & -\left(\frac{1}{2}+\chi\right) h \mathbf{M}-\frac{1}{12} h^{2} \mathbf{C}
\end{array}\right)\left(\begin{array}{c}
\mathbf{u}_{n+1} \\
\mathbf{v}_{n+1}
\end{array}\right) \\
=\left(\begin{array}{cc}
\mathbf{C}-\left(\frac{1}{2}-\chi\right) h \mathbf{K} & \mathbf{M}-\left(\frac{1}{12}-\frac{1}{2} \chi\right) h^{2} \mathbf{K} \\
\mathbf{M}-\left(\frac{1}{12}-\frac{1}{2} \chi\right) h^{2} \mathbf{K} & \left(\frac{1}{2}-\chi\right) h \mathbf{M}-\frac{1}{12} h^{2} \mathbf{C}
\end{array}\right)\left(\begin{array}{c}
\mathbf{u}_{n} \\
\mathbf{v}_{n}
\end{array}\right)+\left(\begin{array}{c}
\frac{1}{2} h \overline{\mathbf{f}} \\
-\frac{1}{12} h^{2} \Delta \mathbf{f}
\end{array}\right)
\end{gathered}
$$

to march it in time with timestep $h$ and forcing terms $\overline{\mathbf{f}}:=(1 / h) \int_{t_{n}}^{t_{n+1}} \mathbf{f}(t) \mathrm{d} t, \Delta \mathbf{f}:=$ $\mathbf{f}_{n+1}-\mathbf{f}_{n}$. The algorithmic parameter $\chi \in[0,1 / 6]$ is related to the scheme spectral radius at infinite frequency via

$$
\chi=\frac{1-\rho_{\infty}}{6\left(1+\rho_{\infty}\right)} \quad \longleftrightarrow \quad \rho_{\infty}=\frac{1-6 \chi}{1+6 \chi}
$$

and therefore controls the numerical dissipation introduced by the integration scheme. The scheme is energy conservative when $\chi=0$ and dissipative for $\chi>0$. Its detailed analysis, on the basis of the scalar model oscillator in its undamped/unforced form, can be found in the original paper [19].

Figure 1 shows the dependency of scaled errors $e_{1} / h, e_{2} / h$ on the timestep for several configurations of the scalar model oscillator, under zero-phase non-resonant sinusoidal excitation, $a(t)=\sin \omega t, \omega \neq \omega_{0}$. Because analytical developments are too tedious in this case, they have been obtained numerically from application of equations (11), (12) to the scheme defined in equation (37). Log-log scaling has been used for the plotting of 
the numerical results. In particular, the influences of the structural $(\zeta \in\{0.0,0.1\})$ and numerical $\left(\rho_{\infty} \in\{0.5,1.0\}\right)$ dampings are shown, as well as that of the accuracy with which the average forcing term $\overline{\mathbf{f}}$ is approximated. For the latter point, the trapezoidal average value, $\overline{\mathbf{f}} \simeq\left(\mathbf{f}_{n}+\mathbf{f}_{n+1}\right) / 2$, and the Simpson-Cavalieri approximation [5, p. 377], $\overline{\mathbf{f}} \simeq\left(\mathbf{f}_{n}+4 \mathbf{f}_{n+1 / 2}+\mathbf{f}_{n+1}\right) / 6$, where $\mathbf{f}_{n+1 / 2}:=\mathbf{f}\left(t_{n}+h / 2\right)$ have been used; these, respectively, provide second-order and fourth-order approximations to the integral. The results are further synthesized in Table I. They give rise to two main comments.

- Although the derivation of the scheme is partially based on the assumption of linear time variation of the displacement, velocity and external forcing fields [19, p. 599], that assumption should not be propagated in the evaluation of the average forcing term entering it. Indeed, in the conservative setting $\left(\rho_{\infty}=1\right)$, the overall scheme order of accuracy drops by 1 , from $k=4$ to $k=3$, when the integral definition of the average forcing is evaluated with second-order accuracy rather than fourth-order.

- In the dissipative setting $\left(\rho_{\infty}<1\right)$, the scheme is second-order $\left(k_{1}=3, k_{2}=2\right)$ and first-order $\left(k_{1}=1, k_{2}=2\right)$ accurate in the absence $(\zeta=0)$ and presence $(\zeta>0)$ of structural damping, respectively. The limited accuracy can be associated with a loss of accuracy of both the forced and homogeneous responses, with the latter being observed in the presence of structural damping only. These observations indicate that (i) the matrices $\mathbf{H}_{0}, \mathbf{H}_{1}$ should include terms coupling the structural and numerical dampings, i.e., terms proportional to $\chi \mathbf{C}$, and (ii) the load vector $\ell_{n}^{n+1}$ should also have a dependency on the numerical damping through parameter $\chi$.

While the first comment clarifies the text of Krenk in regards to the evaluation of the average forcing term, the latter is another clear evidence that the LTE order of the undamped/unforced system should not be extrapolated to more general cases, without any careful study. Indeed, high-order accuracy is not achieved by the scheme, as is, in the presence of structural damping or external forcing, in the numerically dissipative setting.

This example highlights the need for a consistent procedure to evaluate the local truncation error of time integration schemes that includes natural dissipation and forcing in the linear model problem, a recommendation already formulated some 30 years ago by Wood [20]. Recovery of the expected accuracy for this scheme is nevertheless possible through ad hoc modifications to the scheme. These are presented in the next sections.

\section{A FAMILY OF FOUR-LEVEL ONE-STEP SCHEMES}

In their review paper [26], Bottasso and Trainelli make a parallel between three integration schemes, namely the linear time discontinuous Galerkin method (TDG) [17, 32], the dissipative midpoint scheme (ArRo) proposed by Armero and Romero [8] and the parametrized TDG-like scheme (BoTr) introduced by Bottasso and Trainelli [26]. Their introduction and analysis considers the undamped/unforced scalar model problem. We 
Table I. Summary of the accuracy levels observed for Krenk's scheme, using the proposed framework. The scheme expected order of accuracy, as per [19], is only observed in the numerically conservative setting $\left(\rho_{\infty}=1\right)$ with high-order evaluation of the average forcing term. Lower accuracy orders are observed in other configurations.

\begin{tabular}{lcccc}
\hline$\overline{\mathbf{f}}$ accuracy & \multicolumn{2}{c}{$2^{\text {nd }}$ order } & \multicolumn{2}{c}{$4^{\text {th }}$ order } \\
& $\zeta=0$ & $\zeta>0$ & $\zeta=0$ & $\zeta>0$ \\
\hline$\rho_{\infty}=1$ & $k_{1}=4$ & $k_{1}=4$ & $k_{1}=4$ & $k_{1}=4$ \\
& $k_{2}=3$ & $k_{2}=3$ & $k_{2}=4$ & $k_{2}=4$ \\
$\min \left(k_{1}, k_{2}\right)$ & $k=3$ & $k=3$ & $k=4$ & $k=4$ \\
\hline$\rho_{\infty}<1$ & $k_{1}=3$ & $k_{1}=1$ & $k_{1}=3$ & $k_{1}=1$ \\
& $k_{2}=2$ & $k_{2}=2$ & $k_{2}=2$ & $k_{2}=2$ \\
$\min \left(k_{1}, k_{2}\right)$ & $k=2$ & $k=1$ & $k=2$ & $k=1$ \\
\hline
\end{tabular}

extend their comparison in the present section, on the basis of the equation of structural dynamics (1); that is, including structural damping and external forcing for the multiple degrees of freedom model.

\subsection{Linear Time Discontinuous Galerkin scheme - TDG}

The spirit of discontinuous Galerkin (DG) methods is to allow jump discontinuities of the state variables at element connexions (be they in space or in time) by considering a left and a right values at each node. The link between the two values is established by weak enforcement of element boundary conditions in the DG weak form. The jump discontinuity at mesh nodes usually results in numerical stabilization via energy dissipation.

Let us define the average value over the timestep $\bar{x}:=\frac{1}{2}\left(x_{n+1}+x_{n}\right)$, the field variation over the timestep $\Delta x:=x_{n+1}-x_{n}$, the right time limit $x_{n^{+}}:=\lim _{\epsilon \rightarrow 0^{+}} x\left(t_{n}+\epsilon\right)=x\left(t_{n}^{+}\right)$and the jump discontinuity at a given time instant $\llbracket x_{n} \rrbracket:=x_{n^{+}}-x_{n}$. In residual form, the 2 -field weak form relative to the TDG method reads

$$
\begin{aligned}
R_{n} & \equiv \int_{t_{n}}^{t_{n+1}} \mathbf{w}_{1 h} \cdot\left(\mathbf{M} \dot{\mathbf{v}}_{h}+\mathbf{C} \mathbf{v}_{h}+\mathbf{K} \mathbf{u}_{h}-\mathbf{f}\right) \mathrm{d} t+\int_{t_{n}}^{t_{n+1}} \mathbf{w}_{2 h} \cdot\left(\dot{\mathbf{u}}_{h}-\mathbf{v}_{h}\right) \mathrm{d} t \\
& +\mathbf{w}_{1 h}\left(t_{n}^{+}\right) \cdot \mathbf{M} \llbracket \mathbf{v}_{h}\left(t_{n}\right) \rrbracket+\mathbf{w}_{2 h}\left(t_{n}^{+}\right) \cdot \llbracket \mathbf{u}_{h}\left(t_{n}\right) \rrbracket=0 .
\end{aligned}
$$

The subscript $h$ is used to emphasize the finite dimensionality of the fields. Choosing the test functions $\mathbf{w}_{1 h}(t), \mathbf{w}_{2 h}(t)$ and unknown fields $\mathbf{u}_{h}(t), \mathbf{v}_{h}(t)$ to be linear polynomials of type

$$
x(t)=\frac{t_{n+1}-t}{h} x_{n^{+}}+\frac{t-t_{n}}{h} x_{n+1}
$$


with unknown nodal values at $t_{n}^{+}$and $t_{n+1}$, the following update scheme is obtained $[17,32]$

$$
\left(\begin{array}{cccc}
\frac{1}{2} \mathbf{I} & \frac{1}{2} \mathbf{I} & -\frac{h}{3} \mathbf{I} & -\frac{h}{6} \mathbf{I} \\
-\frac{1}{2} \mathbf{I} & \frac{1}{2} \mathbf{I} & -\frac{h}{6} \mathbf{I} & -\frac{h}{3} \mathbf{I} \\
\frac{h}{3} \mathbf{K} & \frac{h}{6} \mathbf{K} & \frac{1}{2} \mathbf{M}+\frac{h}{3} \mathbf{C} & \frac{1}{2} \mathbf{M}+\frac{h}{6} \mathbf{C} \\
\frac{h}{6} \mathbf{K} & \frac{h}{3} \mathbf{K} & -\frac{1}{2} \mathbf{M}+\frac{h}{6} \mathbf{C} & \frac{1}{2} \mathbf{M}+\frac{h}{3} \mathbf{C}
\end{array}\right)\left(\begin{array}{c}
\mathbf{u}_{n^{+}} \\
\mathbf{u}_{n+1} \\
\mathbf{v}_{n^{+}} \\
\mathbf{v}_{n+1}
\end{array}\right)=\left(\begin{array}{c}
\mathbf{u}_{n} \\
\mathbf{0} \\
\mathbf{F}_{1}+\mathbf{M} \mathbf{v}_{n} \\
\mathbf{F}_{2}
\end{array}\right)
$$

Forcing terms $\mathbf{F}_{1}$ and $\mathbf{F}_{2}$ are related to the integrated action of the external loads by

$$
\mathbf{F}_{1}=\int_{t_{n}}^{t_{n+1}} \frac{t_{n+1}-t}{h} \mathbf{f}(t) \mathrm{d} t, \quad \mathbf{F}_{2}=\int_{t_{n}}^{t_{n+1}} \frac{t-t_{n}}{h} \mathbf{f}(t) \mathrm{d} t .
$$

Upon linearly combining the rows of block matrix equation (41), the TDG scheme can be rearranged as follows

$$
\begin{gathered}
\left(\begin{array}{cccc}
\frac{1}{2} \mathbf{K} & \frac{1}{h} \mathbf{M}+\frac{1}{2} \mathbf{C} & \frac{1}{2} \mathbf{K} & \frac{1}{2} \mathbf{C} \\
\mathbf{I} & -\frac{h}{2} \mathbf{I} & \mathbf{0} & -\frac{h}{2} \mathbf{I} \\
\mathbf{0} & \frac{h}{6} \mathbf{I} & \mathbf{I} & -\frac{h}{6} \mathbf{I} \\
-\frac{1}{6} \mathbf{K} & -\frac{1}{6} \mathbf{C} & \frac{1}{6} \mathbf{K} & \frac{1}{h} \mathbf{M}+\frac{1}{6} \mathbf{C}
\end{array}\right)\left(\begin{array}{c}
\mathbf{u}_{n+1} \\
\mathbf{v}_{n+1} \\
\mathbf{u}_{n^{+}} \\
\mathbf{v}_{n^{+}}
\end{array}\right) \\
=\left(\begin{array}{cc}
\mathbf{0} & \frac{1}{h} \mathbf{M} \\
\mathbf{I} & \mathbf{0} \\
\mathbf{I} & \mathbf{0} \\
\mathbf{0} & \frac{1}{h} \mathbf{M}
\end{array}\right)\left(\begin{array}{c}
\mathbf{I}_{1} \\
\mathbf{0} \\
\mathbf{v}_{n}
\end{array}\right)+\left(\begin{array}{c}
\mathbf{0} \\
\frac{2}{h^{2}}\left(t_{n+1 / 2} \mathbf{I}_{1}-\mathbf{I}_{2}\right)
\end{array}\right) .
\end{gathered}
$$

Time $t_{n+1 / 2}:=\frac{1}{2}\left(t_{n+1}+t_{n}\right)$ corresponds to the midstep time. The first row of block matrix equation (43) is related to the equation of motion, the second to the velocity update, the third and four to the update of the internal variables $\mathbf{u}_{n^{+}}, \mathbf{v}_{n^{+}}$, respectively. Integral actions $\mathbf{I}_{1}$ and $\mathbf{I}_{2}$ now replace $\mathbf{F}_{1}$ and $\mathbf{F}_{2}$. They are defined as follows

$$
\mathbf{I}_{\mathbf{1}}=\int_{t_{n}}^{t_{n+1}} \mathbf{f}(t) \mathrm{d} t, \quad \mathbf{I}_{2}=\int_{t_{n}}^{t_{n+1}} t \mathbf{f}(t) \mathrm{d} t
$$

and can be interpreted as time-force moments. Indeed, action $\mathbf{I}_{1}$ defines the force impulse over the timestep and is proportional to the average force over that step, and the combination $\left(t_{n+1 / 2} \mathbf{I}_{1}-\mathbf{I}_{2}\right)$ is nothing else than the opposite of the first-order centered moment over the timestep. These moments capture the time variation of the external loading over the timestep. They are necessary for the scheme to achieve high-order accuracy.

As was illustrated in Section 2.4, the use of low order quadrature for the evaluation of integral terms can result in a decrease of the overall scheme order of accuracy through a loss 
of accuracy on the forced response. For the TDG scheme to achieve third-order accuracy on linear problems, integral terms $\mathbf{I}_{1}, \mathbf{I}_{2}$ should be evaluated analytically or with high order numerical quadrature. In that respect, all formulas achieving third degree exactness provide the sufficient accuracy, e.g., Simpson-Cavalieri, 2-point Gauss-Legendre, cubic Hermite interpolants.

In addition to its high accuracy, the TDG scheme has zero spectral radius at infinite frequency $\rho_{\infty}=0$ (spectral annihilation); high frequency oscillations are nearly reduced to zero in one timestep. Figure $2(\alpha=1 / 6)$ shows the evolution of the spectral radius of the TDG scheme amplification matrix.

To establish the variation of the mechanical energy $(\Delta E)$ over a timestep, i.e., the sum of the variations of the kinetic $(\Delta T)$ and potential $(\Delta V)$ energies over a timestep $\Delta E:=\Delta T+\Delta V$, we form the scalar products of the equation of motion with $\Delta \mathbf{u}$ and of the velocity update with $\mathbf{M} \Delta \mathbf{v}$, and add them

$$
\Delta E=\frac{1}{h} \Delta \mathbf{u} \cdot \mathbf{I}_{1}-\frac{1}{h} \Delta \mathbf{u} \cdot \mathbf{C} \Delta \mathbf{u}-\frac{1}{2} \Delta \mathbf{v} \cdot \mathbf{M} \llbracket \mathbf{v}_{n} \rrbracket-\frac{1}{2} \Delta \mathbf{u} \cdot \mathbf{K} \llbracket \mathbf{u}_{n} \rrbracket .
$$

Noting that $\Delta x=x_{n+1}-x_{n^{+}}+\llbracket x_{n} \rrbracket$ and exploiting the update equations for the internal state variables, the variation of energy can be brought to the following form

$$
\Delta E=\underbrace{\frac{1}{h} \Delta \mathbf{u} \cdot \mathbf{I}_{1}+\frac{6}{h^{2}} \llbracket \mathbf{u}_{n} \rrbracket \cdot\left(t_{n+1 / 2} \mathbf{I}_{1}-\mathbf{I}_{2}\right)}_{\text {work of external forces }}-\underbrace{\frac{1}{h} \Delta \mathbf{u} \cdot \mathbf{C} \Delta \mathbf{u}}_{\begin{array}{c}
\text { viscous } \\
\text { dissipation }
\end{array}}-\underbrace{\left(D_{K}+D_{C}+D_{M}\right),}_{\begin{array}{c}
\text { numerical } \\
\text { dissipation }
\end{array}}
$$

with

$$
D_{K}:=\frac{1}{2} \llbracket \mathbf{u}_{n} \rrbracket \cdot \mathbf{K} \llbracket \mathbf{u}_{n} \rrbracket, \quad D_{C}:=\frac{6}{2 h} \llbracket \mathbf{u}_{n} \rrbracket \cdot \mathbf{C} \llbracket \mathbf{u}_{n} \rrbracket, \quad D_{M}:=\frac{1}{2} \llbracket \mathbf{v}_{n} \rrbracket \cdot \mathbf{M} \llbracket \mathbf{v}_{n} \rrbracket .
$$

It readily appears that jump discontinuities are responsible for numerical damping through energy dissipation, since the stiffness and damping matrices are positive semi-definite, $\mathbf{K}, \mathbf{C} \geq 0$, and the mass matrix positive definite, $\mathbf{M}>0$. This guarantees the unconditional stability of the scheme, even in the absence of mechanical damping (which also contributes to energy dissipation), as the mechanical energy remains bounded. It is also seen that the work of the external forces includes both a continuous contribution proportional to the average forces over the timestep $\left(\mathbf{I}_{1}\right)$ and a discrete contribution associated with the centered time-force moment over the timestep $\left(t_{n+1 / 2} \mathbf{I}_{1}-\mathbf{I}_{2}\right)$.

Further details about this formulation can be found, among other texts, in [17, 26, 32, $37,38]$.

\subsection{Armero and Romero's scheme - ArRo}

Armero and Romero [8] have proposed an energy decaying time integration scheme for application to nonlinear elastodynamics that degenerates into a TDG-like scheme when applied to a linear problem. Their reasoning follows from the introduction of dissipative terms in the equations of motion and the velocity update formula so that they ensure the 
scheme to enjoy the property of algorithmic energy decay by construction. The scheme is second-order accurate. It is parametrized by an algorithmic variable $\alpha \in[0,1 / 6]$ that controls the cut-off frequency at which the spectral radius associated with the linear undamped/unforced oscillator starts its dip toward 0. The scheme exhibits the property of spectral annihilation as well, whatever $\alpha \in(0,1 / 6]$. It is conservative for $\alpha=0$.

Application of the scheme to the problem of linear structural dynamics yields the one-step update equations

$$
\begin{gathered}
\left(\begin{array}{cccc}
\frac{1}{2} \mathbf{K} & \frac{1}{h} \mathbf{M}+\frac{1}{2} \mathbf{C} & \frac{1}{2} \mathbf{K} & \frac{1}{2} \mathbf{C} \\
\mathbf{I} & -\frac{h}{2} \mathbf{I} & \mathbf{0} & -\frac{h}{2} \mathbf{I} \\
\mathbf{0} & \alpha h \mathbf{I} & \mathbf{I} & -\alpha h \mathbf{I} \\
-\alpha \mathbf{K} & -\alpha \mathbf{C} & \alpha \mathbf{K} & \frac{1}{h} \mathbf{M}+\alpha \mathbf{C}
\end{array}\right)\left(\begin{array}{c}
\mathbf{u}_{n+1} \\
\mathbf{v}_{n+1} \\
\mathbf{u}_{n^{+}} \\
\mathbf{v}_{n^{+}}
\end{array}\right)= \\
\left(\begin{array}{cc}
\mathbf{0} & \frac{1}{h} \mathbf{M} \\
\mathbf{I} & \mathbf{0} \\
\mathbf{I} & \mathbf{0} \\
\mathbf{0} & \frac{1}{h} \mathbf{M}
\end{array}\right)\left(\begin{array}{c}
\mathbf{u}_{n} \\
\mathbf{v}_{n}
\end{array}\right)+\left(\begin{array}{c}
\frac{1}{2}\left(\mathbf{f}_{n+1}+\mathbf{f}_{n}\right) \\
\mathbf{0} \\
\mathbf{0} \\
\frac{1}{2}\left(\mathbf{f}_{n}-\mathbf{f}_{n+1}\right)
\end{array}\right)
\end{gathered}
$$

Details about the scheme derivation and its spectral analysis for the undamped/unforced case are to be found in the paper by Armero and Romero [8]. The evolution of the spectral radius as well as these of the relative period error and of the numerical damping are shown in Figure 2 for $\alpha \in\left\{0,(1 / 6)^{3},(1 / 6)^{2}, 1 / 6\right\}$.

Noting that, under the assumption of linear variation, integral actions approximate to

$$
\begin{gathered}
\mathbf{I}_{1}=\int_{t_{n}}^{t_{n+1}} \mathbf{f}(t) \mathrm{d} t \simeq \frac{h}{2}\left(\mathbf{f}_{n+1}+\mathbf{f}_{n}\right), \\
t_{n+1 / 2} \mathbf{I}_{1}-\mathbf{I}_{2}=\int_{t_{n}}^{t_{n+1}}\left(t_{n+1 / 2}-t\right) \mathbf{f}(t) \mathrm{d} t \simeq-\frac{h^{2}}{12}\left(\mathbf{f}_{n+1}-\mathbf{f}_{n}\right),
\end{gathered}
$$

with second-order accuracy, the similitude with the TDG scheme readily appears for $\alpha=1 / 6$; the update structure of the TDG scheme is recovered, but for the loading terms that are evaluated with low order quadrature.

The energy variation over a timestep for the unforced/undamped case can be found in [26]. Extension to the damped/forced case is straightforward from results (46)-(47) by exploitation of the similitude with the TDG scheme

$$
\Delta E=\underbrace{\frac{1}{2} \Delta \mathbf{u} \cdot\left(\mathbf{f}_{n}+\mathbf{f}_{n+1}\right)-\frac{1}{12 \alpha} \llbracket \mathbf{u}_{n} \rrbracket \cdot\left(\mathbf{f}_{n+1}-\mathbf{f}_{n}\right)}_{\text {work of external forces }}-\underbrace{\frac{1}{h} \Delta \mathbf{u} \cdot \mathbf{C} \Delta \mathbf{u}}_{\begin{array}{c}
\text { viscous } \\
\text { dissipation }
\end{array}}-\underbrace{\left(D_{K}+D_{C}+D_{M}\right),}_{\begin{array}{c}
\text { numerical } \\
\text { dissipation }
\end{array}}
$$



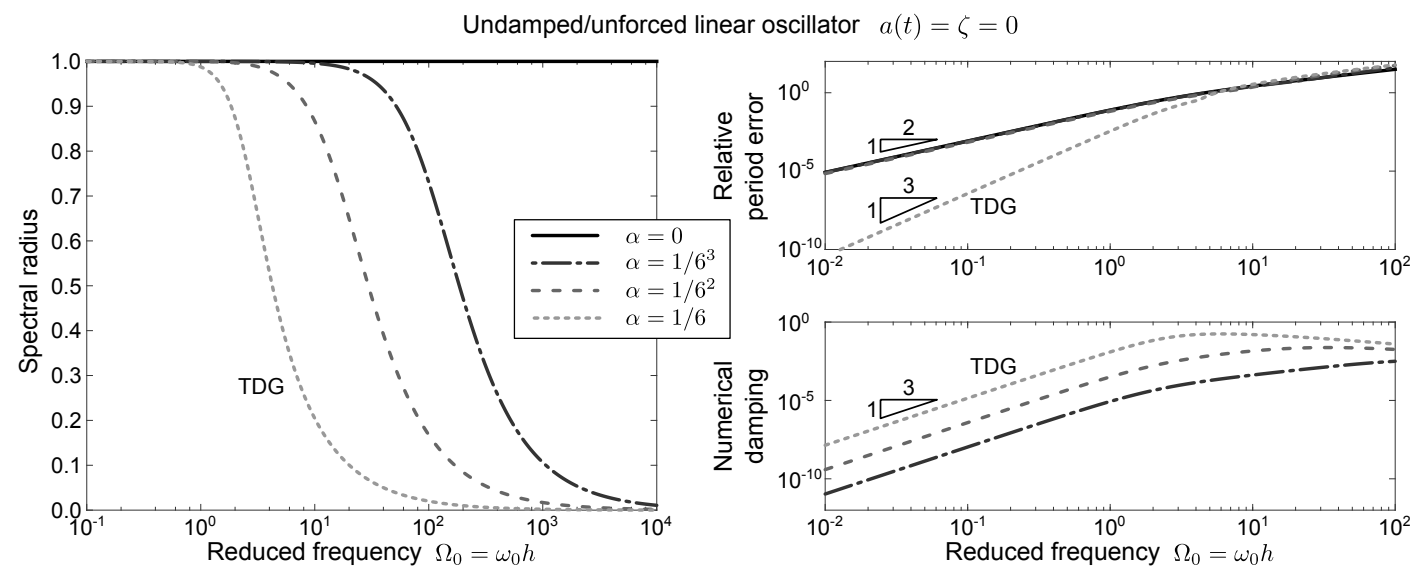

Figure 2. The left plot shows the variation of the ArRo scheme spectral radius when applied to the scalar undamped/unforced linear oscillator. Algorithmic parameter $\alpha$ controls the cutoff frequency at which damping becomes significant. The spectral radius at infinite frequency is zero, $\rho_{\infty}=0$, whatever $\alpha \in(0,1 / 6]$. The case $\alpha=0$ corresponds to numerically conservative integration $\left(\rho_{\infty}=1\right)$. The right plot depicts the relative period error and the numerical damping arising from the numerical integration.

with

$$
D_{K}:=\frac{1}{2} \llbracket \mathbf{u}_{n} \rrbracket \cdot \mathbf{K} \llbracket \mathbf{u}_{n} \rrbracket, \quad D_{C}:=\frac{1}{2 \alpha h} \llbracket \mathbf{u}_{n} \rrbracket \cdot \mathbf{C} \llbracket \mathbf{u}_{n} \rrbracket, \quad D_{M}:=\frac{1}{2} \llbracket \mathbf{v}_{n} \rrbracket \cdot \mathbf{M} \llbracket \mathbf{v}_{n} \rrbracket .
$$

Dissipative factors play a role similar to the one they have in the case of the TDG scheme, for $\alpha>0$. For $\alpha=0$, the ArRo scheme degenerates into the conservative second-order midpoint scheme. Jump discontinuities vanish $\left(\mathbf{u}_{n^{+}}=\mathbf{u}_{n}, \mathbf{v}_{n^{+}}=\mathbf{v}_{n}\right)$ and the mechanical energy variation over a timestep simplifies to

$$
\Delta E=\underbrace{\frac{1}{2} \Delta \mathbf{u} \cdot\left(\mathbf{f}_{n}+\mathbf{f}_{n+1}\right)}_{\begin{array}{c}
\text { work of } \\
\text { ext. forces }
\end{array}}-\underbrace{\frac{1}{h} \Delta \mathbf{u} \cdot \mathbf{C} \Delta \mathbf{u} .}_{\begin{array}{c}
\text { viscous } \\
\text { dissipation }
\end{array}}
$$

The scheme is thus numerically conservative for $\alpha=0$. Furthermore, it is unconditionally stable for $\alpha \geq 0$.

\subsection{Bottasso and Trainelli's scheme - BoTr}

For some applications, spectral annihilation might not be a desired property for an integration scheme. To gain control on the spectral radius at infinite frequency and, thereby, on the amount of numerical dissipation in the high-frequency range, Bottasso and 
Trainelli [26] have proposed another variation of the TDG scheme. It is given by

$$
\begin{gathered}
\left(\begin{array}{cccc}
\frac{1}{2} \mathbf{K} & \frac{1}{h} \mathbf{M}+\frac{1}{2} \mathbf{C} & \frac{1}{2} \mathbf{K} & \frac{1}{2} \mathbf{C} \\
\mathbf{I} & -\frac{h}{2} \mathbf{I} & \mathbf{0} & -\frac{h}{2} \mathbf{I} \\
\mathbf{0} & \frac{h}{6} \mathbf{I} & \mathbf{I} & -\beta \frac{h}{6} \mathbf{I} \\
-\frac{1}{6} \mathbf{K} & -\frac{1}{6} \mathbf{C} & \frac{\beta}{6} \mathbf{K} & \frac{1}{h} \mathbf{M}+\frac{\beta}{6} \mathbf{C}
\end{array}\right)\left(\begin{array}{c}
\mathbf{u}_{n+1} \\
\mathbf{v}_{n+1} \\
\mathbf{u}_{n^{+}} \\
\mathbf{v}_{n^{+}}
\end{array}\right) \\
=\left(\begin{array}{cc}
\mathbf{0} & \frac{1}{h} \mathbf{M} \\
\frac{1}{h} \mathbf{I}_{1} \\
\mathbf{0} \\
\mathbf{I} & \mathbf{0} \\
\mathbf{I} & (1-\beta) \frac{h}{6} \mathbf{I} \\
\frac{\beta-1}{6} \mathbf{K} & \frac{1}{h} \mathbf{M}+\frac{\beta-1}{6} \mathbf{C}
\end{array}\right)\left(\begin{array}{c}
\mathbf{u}_{n} \\
\mathbf{v}_{n}
\end{array}\right)+\left(\begin{array}{c}
\frac{2}{h^{2}}\left(t_{n+1 / 2} \mathbf{I}_{1}-\mathbf{I}_{2}\right)
\end{array}\right) .
\end{gathered}
$$

Similarly to the TDG scheme, integral actions $\mathbf{I}_{1}, \mathbf{I}_{2}$ should be evaluated with third degree exactness. Algorithmic parameter $\beta \in[0,1]$ enables the control of the spectral radius at infinity via

$$
\beta=\frac{1-\rho_{\infty}}{1+\rho_{\infty}} \longleftrightarrow \rho_{\infty}=\frac{1-\beta}{1+\beta} .
$$

Its influence on the scheme properties, in the undamped/unforced case, is depicted in Figure 3. The scheme is third-order accurate in the dissipative setting $(\beta \in(0,1])$ and fourth-order accurate in the numerically conservative setting $(\beta=0)$. The limit case $\beta=1$ corresponds to the TDG scheme.

Parameter $\beta$ also plays a direct role in the numerical dissipation during timestepping, as it explicitly appears in the mechanical energy variation over a timestep

$$
\Delta E=\underbrace{\frac{1}{h} \Delta \mathbf{u} \cdot \mathbf{I}_{1}+\frac{6}{h^{2}} \llbracket \mathbf{u}_{n} \rrbracket \cdot\left(t_{n+1 / 2} \mathbf{I}_{1}-\mathbf{I}_{2}\right)}_{\text {work of external forces }}-\underbrace{\frac{1}{h} \Delta \mathbf{u} \cdot \mathbf{C} \Delta \mathbf{u}}_{\begin{array}{c}
\text { viscous } \\
\text { dissipation }
\end{array}}-\underbrace{\left(\beta D_{K}+D_{C}+\beta D_{M}\right),}_{\begin{array}{c}
\text { numerical } \\
\text { dissipation }
\end{array}}
$$

with the dissipative potentials given in equation (47). Stability is thus guaranteed for all timestep values, through the boundedness of the mechanical energy. When $\beta=0$, jump discontinuities vanish $\left(\mathbf{u}_{n^{+}}=\mathbf{u}_{n}, \mathbf{v}_{n^{+}}=\mathbf{v}_{n}\right)$; the scheme is numerically conservative and the energy variation over a timestep reduces to

$$
\Delta E=\underbrace{\frac{1}{h} \Delta \mathbf{u} \cdot \mathbf{I}_{1}}_{\begin{array}{c}
\text { work of } \\
\text { ext. forces }
\end{array}}-\frac{1}{h} \Delta \mathbf{u} \cdot \mathbf{C} \Delta \mathbf{u} .
$$

Applications of this scheme to problems of the nonlinear flexible multibody class are to be found in $[25,39]$, for which it was originally developed. 

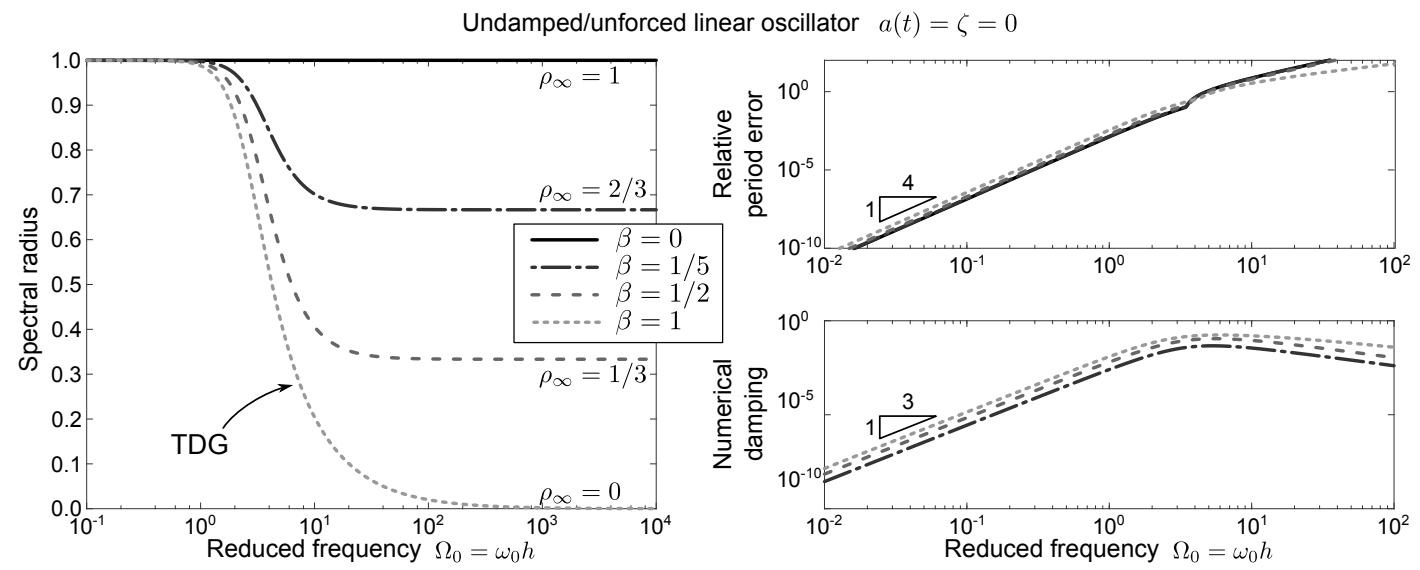

Figure 3. The left plot shows the variation of the BoTr scheme spectral radius when applied to the scalar undamped/unforced linear oscillator. The spectral radius at infinite frequency is adjusted via the algorithmic parameter $\beta=\left(1-\rho_{\infty}\right) /\left(1+\rho_{\infty}\right) \in[0,1]$. The right plot depicts the relative period error and the numerical damping arising from the numerical integration. The TDG scheme is recovered for $\beta=1$. The above plots also characterize the spectral properties of the amplification matrix of Krenk's scheme, for the undamped/unforced configuration.

\subsection{Scheme accuracy}

The framework introduced in Section 2.2 is adapted for two-level one-step integration schemes. The four-level integration schemes presented above need to be recast in their equivalent two-level form before their orders of accuracy can be assessed. To that end, we

eliminate the internal degrees of freedom using static condensation. Rewriting the one-step update system under the partitioned form

$$
\left(\begin{array}{cc}
\mathbf{H}_{0}^{A} & \mathbf{H}_{0}^{B} \\
\mathbf{H}_{0}^{C} & \mathbf{H}_{0}^{D}
\end{array}\right)\left(\begin{array}{c}
\mathbf{x}_{n+1} \\
\mathbf{x}_{n^{+}}
\end{array}\right)=\left(\begin{array}{c}
\mathbf{H}_{1}^{A} \\
\mathbf{H}_{1}^{C}
\end{array}\right) \mathbf{x}_{n}+\left(\begin{array}{c}
\ell_{n}^{n+1, A} \\
\ell_{n}^{n+1, C}
\end{array}\right),
$$

with state (column) vector $\mathbf{x}_{n}=\left[\mathbf{u}_{n} ; \mathbf{v}_{n}\right]$, it is readily established that

$$
\begin{gathered}
\left(\mathbf{H}_{0}^{A}-\mathbf{H}_{0}^{B}\left(\mathbf{H}_{0}^{D}\right)^{-1} \mathbf{H}_{0}^{C}\right) \mathbf{x}_{n+1} \\
=\left(\mathbf{H}_{1}^{A}-\mathbf{H}_{0}^{B}\left(\mathbf{H}_{0}^{D}\right)^{-1} \mathbf{H}_{1}^{C}\right) \mathbf{x}_{n}+\boldsymbol{\ell}_{n}^{n+1, A}-\mathbf{H}_{0}^{B}\left(\mathbf{H}_{0}^{D}\right)^{-1} \boldsymbol{\ell}_{n}^{n+1, C} .
\end{gathered}
$$

The determinant of matrix $\mathbf{H}_{0}^{D}$ is given by

$$
\left|\mathbf{H}_{0}^{D}\right|=\frac{1}{h}\left|\mathbf{M}+\theta h \mathbf{C}+\theta^{2} h^{2} \mathbf{K}\right|,
$$

with $\theta=1 / 6, \theta=\alpha$ and $\theta=\beta / 6$ for the TDG, ArRo and BoTr schemes, respectively. It is positive, $\left|\mathbf{H}_{0}^{D}\right|>0$, given the assumptions of definiteness of the stiffness, damping and mass matrices, which guarantees the invertibility of the matrix. The condensed iteration matrices 

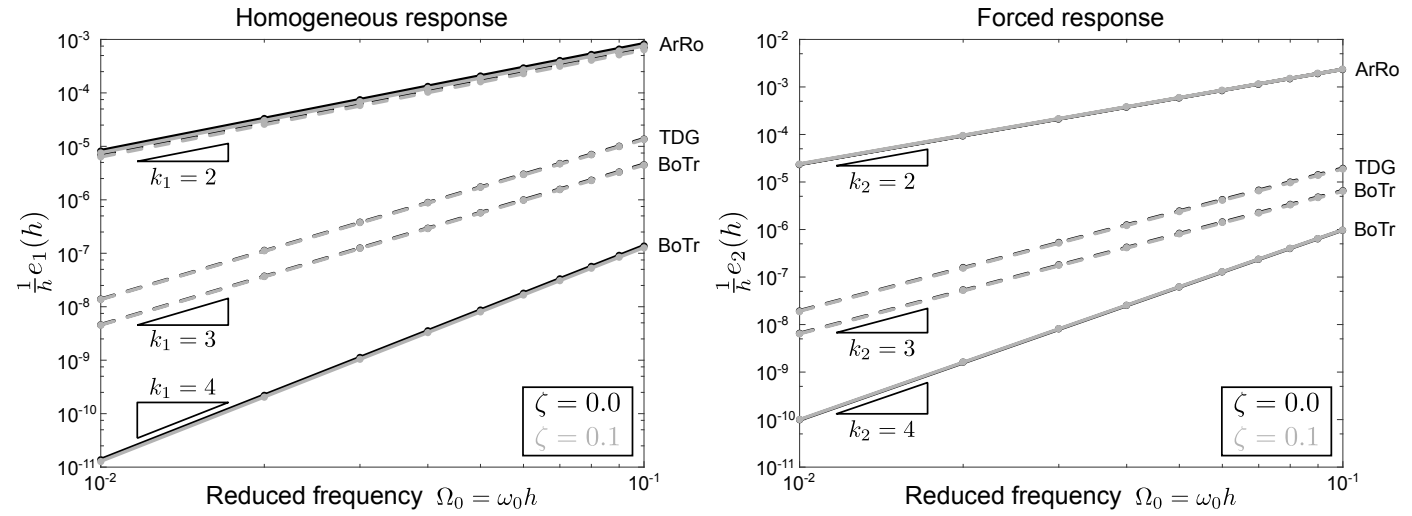

Figure 4. Scaled error dependency on the timestep, comparison of the accuracies achieved by the TDG, ArRo and BoTr schemes on the linear scalar model problem with sinusoidal excitation $(a(t)=\sin \omega t)$ and model parameters $\left(\omega_{0}, \omega\right)=(1,2)$. The left plot corresponds to the scaled error norm on the homogeneous response component $\left(e_{1}(h) / h\right)$ whereas the error on the forced response component $\left(e_{2}(h) / h\right)$ is considered in the right one. Solid lines $(-)$ depict results relative to the conservative setting of the scheme $\left(\rho_{\infty}=1\right)$, dashed ones $(--)$ illustrate the dissipative setting of the scheme (TDG: $\rho_{\infty}=0$, ArRo: $\alpha=1 / 36$, BoTr: $\beta=1 / 3$ ). Black lines correspond to the undamped case $(\zeta=0)$; gray ones to the damped case $(\zeta=0.1)$. Line slopes represent the orders of accuracy $k_{1}, k_{2}$. The observed orders of accuracy $\left(k=\min \left\{k_{1}, k_{2}\right\}\right)$ are consistent with those predicted by the conventional analysis based on the amplification matrix.

and vector are then identified as

$$
\begin{gathered}
\widehat{\mathbf{H}}_{0}=\mathbf{H}_{0}^{A}-\mathbf{H}_{0}^{B}\left(\mathbf{H}_{0}^{D}\right)^{-1} \mathbf{H}_{0}^{C}, \quad \widehat{\mathbf{H}}_{1}=\mathbf{H}_{1}^{A}-\mathbf{H}_{0}^{B}\left(\mathbf{H}_{0}^{D}\right)^{-1} \mathbf{H}_{1}^{C}, \\
\widehat{\boldsymbol{\ell}}_{n}^{n+1}=\boldsymbol{\ell}_{n}^{n+1, A}-\mathbf{H}_{0}^{B}\left(\mathbf{H}_{0}^{D}\right)^{-1} \boldsymbol{\ell}_{n}^{n+1, C} .
\end{gathered}
$$

Computation of scaled errors $e_{1}(h) / h, e_{2}(h) / h$ versus the timestep $h$, on the basis of the condensed scheme, yields the results of Figure 4. A sinusoidal loading is considered; the Cavalieri-Simpson quadrature formula is used to numerically integrate the force actions. Contrary to Krenk's scheme, the accuracy of the schemes is independent of the presence of natural damping and of external loading. Also, the schemes achieve the expected accuracies, i.e., the ones predicted by the error analysis on the undamped/unforced linear oscillator; the TDG scheme is third-order accurate, the ArRo one is second order and the BoTr is fourth-order accurate in the conservative setting $\left(\rho_{\infty}=1\right)$ and third-order accurate in the dissipative one $\left(\rho_{\infty}<1\right)$. The accuracy properties of the schemes are summarized in Table II.

Quite unexpectedly, the performance of the BoTr scheme matches the one expected for Krenk's scheme. In fact, the amplification matrices of both schemes, as computed on the undamped/unforced linear oscillator, can be shown to match exactly under the substitution $\chi=\beta / 6$, a relation that was already hinted by equations (38) and (54). This similarity is exploited in the next section to provide the corrections to Krenk's scheme required to achieve the originally expected performances. 
Table II. Property summary of the TDG-like four-level one-step algorithms introduced in Section 3. Scheme settings that have a unit spectral radius at infinite frequency, $\rho_{\infty}=1$, correspond to energy preserving configurations; others do to algorithmic energy decaying schemes.

\begin{tabular}{lccc}
\hline Scheme & Algo. param. & $\rho_{\infty}$ & Accuracy $(k)$ \\
\hline TDG & $/$ & 0 & 3 \\
ArRo & $\alpha \in(0,1 / 6]$ & 0 & 2 \\
& $\alpha=0$ & 1 & 2 \\
BoTr & $\beta \in(0,1]$ & $\frac{1-\beta}{1+\beta}$ & 3 \\
& $\beta=0$ & 1 & 4 \\
\hline
\end{tabular}

\section{CORRECTION TO KRENK'S SCHEME}

The accuracy analysis of Krenk's scheme has hinted the need for additional terms in the scheme formulation that include the numerical damping parameter $\chi$. In order to identify these terms, we transform the BoTr scheme into its two-level form and exploit its similitude with Krenk's one.

To that end, we reduce formulation (53) using the static condensation formulated in equation (58). With the help of the matrix block inversion formula [40]

$$
\mathbf{S}^{-1}=\left(\begin{array}{cc}
\mathbf{A} & \mathbf{B} \\
\mathbf{C} & \mathbf{D}
\end{array}\right)^{-1}=\left(\begin{array}{cc}
\mathbf{A}^{-1}+\mathbf{A}^{-1} \mathbf{B}(\mathbf{S} \backslash \mathbf{A})^{-1} \mathbf{C A}^{-1} & -\mathbf{A}^{-1} \mathbf{B}(\mathbf{S} \backslash \mathbf{A})^{-1} \\
-(\mathbf{S} \backslash \mathbf{A})^{-1} \mathbf{C A}^{-1} & (\mathbf{S} \backslash \mathbf{A})^{-1}
\end{array}\right),
$$

where $(\mathbf{S} \backslash \mathbf{A})=\mathbf{D}-\mathbf{C A}^{-1} \mathbf{B}$ denotes the Schur complement of the partitioned matrix $\mathbf{S}$ with respect to $\mathbf{A}$, we identify the entries of the reduced iteration matrices and vector

$$
\left(\begin{array}{cc}
\widehat{\mathbf{H}}_{0}^{\mathbf{u u}} & \widehat{\mathbf{H}}_{0}^{\mathbf{u v}} \\
\widehat{\mathbf{H}}_{0}^{\mathbf{v u}} & \widehat{\mathbf{H}}_{0}^{\mathbf{v v}}
\end{array}\right)\left(\begin{array}{c}
\mathbf{u}_{n+1} \\
\mathbf{v}_{n+1}
\end{array}\right)=\left(\begin{array}{cc}
\widehat{\mathbf{H}}_{1}^{\mathbf{u u}} & \widehat{\mathbf{H}}_{1}^{\mathbf{u v}} \\
\widehat{\mathbf{H}}_{1}^{\mathbf{v u}} & \widehat{\mathbf{H}}_{1}^{\mathbf{v v}}
\end{array}\right)\left(\begin{array}{c}
\mathbf{u}_{n} \\
\mathbf{v}_{n}
\end{array}\right)+\left(\begin{array}{c}
\widehat{\boldsymbol{\ell}}_{n}^{n+1, \mathbf{u}} \\
\widehat{\boldsymbol{\ell}}_{n}^{n+1, \mathbf{v}}
\end{array}\right)
$$

Copyright (c) 0000 John Wiley \& Sons, Ltd.

Prepared using nmeauth.cls
Int. J. Numer. Meth. Engng (0000)

DOI: $10.1002 / \mathrm{nme}$ 
as

$$
\begin{aligned}
\widehat{\mathbf{H}}_{0}^{\mathbf{u u}}= & \frac{1}{2} \mathbf{K}+\left(\frac{\beta h}{72} \mathbf{K}+\frac{1}{12} \mathbf{C}\right)(\mathbf{S} \backslash \mathbf{A})^{-1} \mathbf{K}, \\
\widehat{\mathbf{H}}_{0}^{\mathbf{u v}}= & \frac{1}{h} \mathbf{M}+\frac{1}{2} \mathbf{C}-\frac{h}{12} \mathbf{K}+\frac{\beta^{2} h^{2}}{432} \mathbf{K}(\mathbf{S} \backslash \mathbf{A})^{-1} \mathbf{K}+\frac{\beta h}{72}\left(\mathbf{C}(\mathbf{S} \backslash \mathbf{A})^{-1} \mathbf{K}+\mathbf{K}(\mathbf{S} \backslash \mathbf{A})^{-1} \mathbf{C}\right) \\
& +\frac{1}{12} \mathbf{C}(\mathbf{S} \backslash \mathbf{A})^{-1} \mathbf{C}, \\
\widehat{\mathbf{H}}_{0}^{\mathbf{v u}=} & \mathbf{I}-\frac{h}{12}(\mathbf{S} \backslash \mathbf{A})^{-1} \mathbf{K}, \\
\widehat{\mathbf{H}}_{0}^{\mathbf{v v}=}= & -\frac{h}{2} \mathbf{I}-\frac{\beta h^{2}}{72}(\mathbf{S} \backslash \mathbf{A})^{-1} \mathbf{K}-\frac{h}{12}(\mathbf{S} \backslash \mathbf{A})^{-1} \mathbf{C}, \\
\widehat{\mathbf{H}}_{1}^{\mathbf{u u}=}= & \frac{1}{2} \mathbf{K}+\frac{\beta h}{72} \mathbf{K}(\mathbf{S} \backslash \mathbf{A})^{-1} \mathbf{K}+\frac{1}{12} \mathbf{C}(\mathbf{S} \backslash \mathbf{A})^{-1} \mathbf{K}, \\
\widehat{\mathbf{H}}_{1}^{\mathbf{u v}=}= & \frac{1}{h} \mathbf{M}-\frac{1}{2} \mathbf{C}-\frac{h}{12} \mathbf{K}+\frac{\beta^{2} h^{2}}{432} \mathbf{K}(\mathbf{S} \backslash \mathbf{A})^{-1} \mathbf{K}+\frac{\beta h}{72}\left(\mathbf{C}(\mathbf{S} \backslash \mathbf{A})^{-1} \mathbf{K}+\mathbf{K}(\mathbf{S} \backslash \mathbf{A})^{-1} \mathbf{C}\right) \\
& +\frac{1}{12} \mathbf{C}(\mathbf{S} \backslash \mathbf{A})^{-1} \mathbf{C}, \\
\widehat{\mathbf{H}}_{1}^{\mathbf{v u}=}= & \mathbf{I}-\frac{h}{12}(\mathbf{S} \backslash \mathbf{A})^{-1} \mathbf{K}, \\
\widehat{\mathbf{H}}_{1}^{\mathbf{v v}=} & \frac{h}{2} \mathbf{I}-(\mathbf{S} \backslash \mathbf{A})^{-1}\left(\frac{h}{12} \mathbf{C}+\frac{\beta h^{2}}{72} \mathbf{K}\right), \\
\widehat{\boldsymbol{\ell}}_{n}^{n+1, \mathbf{u}}= & \frac{1}{h} \mathbf{I}_{1}-\left(\frac{\beta}{6 h} \mathbf{K}+\frac{1}{h^{2}} \mathbf{C}\right)(\mathbf{S} \backslash \mathbf{A})^{-1}\left(t_{n+1 / 2} \mathbf{I}_{1}-\mathbf{I}_{2}\right), \\
\widehat{\boldsymbol{\ell}}_{n}^{n+1, \mathbf{v}}= & \frac{1}{h}(\mathbf{S} \backslash \mathbf{A})^{-1}\left(t_{n+1 / 2} \mathbf{I}_{1}-\mathbf{I}_{2}\right) .
\end{aligned}
$$

The Schur complement values $\mathbf{S} \backslash \mathbf{A}=\frac{1}{h} \mathbf{M}+\frac{\beta}{6} \mathbf{C}+\frac{\beta^{2} h}{36} \mathbf{K}$ and is invertible in virtue of the definiteness assumptions on the stiffness, damping and mass matrices. Simplifications then follow by left-multiplication of partitioned matrix equation (62) by the conditioning matrix

$$
\mathbf{Q}=\left(\begin{array}{cc}
h \mathbf{I} & \frac{\beta h}{6} \mathbf{K}+\mathbf{C} \\
-\frac{\beta h^{2}}{6} \mathbf{I} & \mathbf{M}
\end{array}\right)
$$

Completing all algebra, we obtain the simplified two-level form of the BoTr scheme

$$
\begin{aligned}
& \left(\begin{array}{cc}
\mathbf{C}+\left(\frac{1}{2}+\frac{\beta}{6}\right) h \mathbf{K} & \mathbf{M}-\left(\frac{1}{12}+\frac{\beta}{12}\right) h^{2} \mathbf{K} \\
\mathbf{M}-\left(\frac{1}{12}+\frac{\beta}{12}\right) h^{2} \mathbf{K} & -\left(\frac{1}{2}+\frac{\beta}{6}\right) h \mathbf{M}-\left(\frac{1}{12}+\frac{\beta}{12}\right) h^{2} \mathbf{C}
\end{array}\right)\left(\begin{array}{c}
\mathbf{u}_{n+1} \\
\mathbf{v}_{n+1}
\end{array}\right) \\
& =\left(\begin{array}{cc}
\mathbf{C}-\left(\frac{1}{2}-\frac{\beta}{6}\right) h \mathbf{K} & \mathbf{M}-\left(\frac{1}{12}-\frac{\beta}{12}\right) h^{2} \mathbf{K} \\
\mathbf{M}-\left(\frac{1}{12}-\frac{\beta}{12}\right) h^{2} \mathbf{K} & \left(\frac{1}{2}-\frac{\beta}{6}\right) h \mathbf{M}-\left(\frac{1}{12}-\frac{\beta}{12}\right) h^{2} \mathbf{C}
\end{array}\right)\left(\begin{array}{c}
\mathbf{u}_{n} \\
\mathbf{v}_{n}
\end{array}\right) \\
& +\left(\begin{array}{c}
\mathbf{I}_{1} \\
\left(t_{n+1 / 2}-\frac{\beta}{6} h\right) \mathbf{I}_{1}-\mathbf{I}_{2}
\end{array}\right) \text {. }
\end{aligned}
$$


Similitude with Krenk's scheme (37) becomes obvious by noting the equivalence $\chi \longleftrightarrow \beta / 6$, that $h \overline{\mathbf{f}}=\mathbf{I}_{1}$ and that, under the assumption of linear evolution of the external load, $-h^{2} \Delta \mathbf{f} / 12=t_{n+1 / 2} \mathbf{I}_{\mathbf{1}}-\mathbf{I}_{2}$.

In particular, the schemes are fully equivalent in the undamped/unforced case. The plots of Figure 3 thus characterize Krenk's scheme as well. For other configurations, however, discrepancies between the two schemes are noted. They reveal the terms missing in Krenk's formulation to ensure full consistency in regards to the introduction of artificial (numerical) damping and confirm the observations to the analysis results given in Figure 1 and Table I.

1. The iteration matrices should include a damping term proportional to the damping matrix and the algorithmic parameter $\chi$ to enable third-order accuracy in the numerically dissipative setting in the presence of structural damping.

2. The first-order time-force moment entering the evolution equations should be evaluated with respect to shifted midstep time $t_{n+(1-\beta) / 2}:=t_{n}+(1-\beta) h / 2$ and not the midstep time $t_{n+1 / 2}$. Whether low-order or high-order quadrature is used to evaluate it, does not alter the order of the scheme; only $C_{2}$ is affected.

The missing terms in the integration scheme given in equation (37) are the result of the constructive nature that underlies the derivation of the scheme, as applied to the undamped/unforced model problem. Had the author accounted for damping and external forcing in the engineering and analysis of the scheme, the accuracy issues would have been identified from the start.

Given that discontinuity variables do not present a physical interest in problems of structural dynamics, the two-level formulation of the BoTr scheme presents several advantages over its four-level original version. First, the reduction of the original four-level system to an equivalent two-level form leads to a reduction of the state-space dimension by a factor two. On large systems, this can result in a significant reduction of the computational burden, even though the two-level form is not as sparse as the four-level one. Second, the iteration matrices of the two-level form are symmetric, contrary to the ones of the four-level form. This symmetry can appropriately be exploited to lessen the required storage space as well as for solving the update system of equations at each increment using an ad hoc procedure. This, in turn, can also prove beneficial as regards the computational effort.

\section{CONCLUSIONS}

This paper introduces a novel approach for the estimation of the local truncation error (LTE) arising in two-level one-step time integration schemes for linear structural dynamics. It naturally accounts for structural damping and external forcing, terms that are generally neglected in conventional accuracy analyses. Moreover, it proposes a separate treatment of the errors relative to the free and forced responses of the linear structural dynamics problem. Subsequent to that presentation is the application of this accuracy analysis framework to two integration schemes. The second example reveals the shortcomings of the traditional accuracy analysis that fails at identifying deficiencies in the analyzed high-order scheme. 
Follow developments for the restoration of the expected high-order accuracy of the analyzed scheme. They imply the reduction of a four-level one-step scheme related to the time discontinuous Galerkin (TDG) method to its equivalent two-level formulation and the identification of missing terms in the original formulation of the high-order scheme.

The estimation of the LTE relative to the use of time integration schemes as applied to linear structural dynamics problems is typically performed on the basis of the scalar undamped homogeneous linear oscillator. The scheme order of accuracy is obtained by spectral analysis of the scheme amplification matrix. By design, this procedure does not account for the effects of natural damping and external loading on the scheme response. It is therefore prone to shortcomings, for it is incomplete. In that sense, the approach we propose is complete for it is based on the generic governing equation of linear structural dynamics, including structural damping and external forcing. It is based on the evaluation of the error norm proposed by Romero [36] that quantifies the LTE in a metric related to the mechanical energy of the error on the displacement and velocity variables. Decomposition of the error in terms of the homogeneous and particular responses is provided to enable their separate treatment. Furthermore, the proposed formulation does not require the uncoupling of the equations of motion through modal expansion. Assessment of the scheme accuracy can therefore be performed in usage conditions with no assumptions on the nature of structural damping.

As illustration examples, we apply the framework to the trapezoidal scheme as well as to the high-order one engineered by Krenk [19]. The analytical analysis of the first example recovers the well-known second-order nature of the trapezoidal scheme. The numerical analysis of the latter, however, brings to light losses of accuracy when the high-order scheme is used in the numerically dissipative setting, in the presence of structural damping or external forcing. The results of the throrough analysis performed by the author, on the basis of the scalar undamped/unforced linear oscillator, are shown to not extend to other configurations of the oscillator. This underscores the importance of the completeness of the accuracy analysis, a point that was already raised by Wood [20] that, nevertheless, has not been of much application in the literature.

In order to correct the deficiencies of the analyzed high-order scheme, we demonstrate its similitude with the BoTr scheme, a TDG-related scheme proposed by Bottasso and Trainelli [26], enabling the identification of the missing terms in the high-order scheme. First, we extend their exposition of TDG-related energy decaying schemes from the scalar undamped/unforced oscillator to the generic model of linear structural dynamics and conduct the accuracy analysis of these schemes using the proposed approach. Unconditional stability, for the linear problem, is also proven on the basis of energy arguments. Second, we recast the four-level BoTr scheme into its equivalent two-level form, by elimination of the internal variables through static condensation. The reduced form of the scheme is then shown equivalent to that of the deficient high-order one, in the absence of numerical damping. We then hypothesize that the two schemes are equivalent for all configurations. Not only does this assumption provide all the corrective terms by identification of the discrepancies between to the two formulations, it also opens the way to making a connection between TDG-related schemes, based on a sound mathematical formulation, and the ones derived 
on the basis of an integration by parts of the equation of motion, for which numerical dissipation is introduced by way of constructive and intuitive arguments.

\section{ACKNOWLEDGEMENTS}

The authors thank the reviewer for his careful critique of the original manuscript and the ensuing suggestions. The partial support of the Graduate School of the University of Minnesota, through a Doctoral Dissertation Fellowship, is gratefully acknowledged.

References

1. Hilber HM, Hughes TJR. Collocation, dissipation and overshoot for time integration schemes in structural dynamics. Earthquake Engineering $\&$ Structural Dynamics 1978; 6(1):99-117, doi: 10.1002 /eqe.4290060111.

2. Dahlquist GG. A special stability problem for linear multistep methods. BIT Numerical Mathematics 1963; 3(1):27-43, doi:10.1007/BF01963532.

3. Hulbert GM. Computational Structural Dynamics, chap. 5. John Wiley \& Sons, Ltd, 2004, doi: 10.1002/0470091355.ecm028.

4. Zhou X, Tamma KK. Design, analysis, and synthesis of generalized single step single solve and optimal algorithms for structural dynamics. International Journal for Numerical Methods in Engineering 2004; 59(5):597-668, doi:10.1002/nme.873.

5. Quarteroni A, Sacco R, Saleri F. Numerical Mathematics. Texts in Applied Mathematics, SpringerVerlag New York, Inc., 2010.

6. Ortiz M. A note on energy conservation and stability of nonlinear time-stepping algorithms. Computers \& Structures 1986; 24(1):167-168, doi:10.1016/0045-7949(86)90346-9.

7. Simo J, Tarnow N. The discrete energy-momentum method. Conserving algorithms for nonlinear elastodynamics. Zeitschrift für angewandte Mathematik und Physik ZAMP 1992; 43(5):757-792, doi:10.1007/BF00913408.

8. Armero F, Romero I. On the formulation of high-frequency dissipative time-stepping algorithms for nonlinear dynamics. Part II: second-order methods. Computer Methods in Applied Mechanics and Engineering 2001; 190:6783-6824, doi:10.1016/S0045-7825(01)00233-X.

9. Bottasso CL, Borri M. Integrating finite rotations. Computer Methods in Applied Mechanics and Engineering 1998; 164(3-4):307-331, doi:10.1016/S0045-7825(98)00031-0.

10. Krenk S. Energy conservation in Newmark based time integration algorithms. Computer Methods in Applied Mechanics and Engineering 2006; 195(44-47):6110-6124, doi:10.1016/j.cma.2005.12.001.

11. Clough R, Penzien J. Dynamics of structures. Dynamics of Structures Ray W. Clough, Joseph Penzien, McGraw-Hill Book Co., 1975.

12. Géradin M, Rixen D. Mechanical Vibrations: Theory and Application to Structural Dynamics. Wiley, 1997.

13. Hughes TJR, Belytschko T. A Précis of Developments in Computational Methods for Transient Analysis. Journal of Applied Mechanics-transactions of The Asme 1983; 50:1033-1041, doi: 10.1115/1.3167186.

14. Hughes TJR. The finite element method: linear static and dynamic finite element analysis. Dover, 1987.

15. Chung J, Hulbert GM. A Time Integration Algorithm for Structural Dynamics With Improved Numerical Dissipation: The Generalized-alpha Method. Journal of Applied Mechanics-transactions of The Asme 1993; 60:371-375, doi:10.1115/1.2900803.

16. Wood WL. On the effect of natural damping on the stability of a time-stepping scheme. Communications in Applied Numerical Methods 1987; 3(2):141-144, doi:10.1002/cnm.1630030210.

17. Hulbert GM. Time finite element methods for structural dynamics. International Journal for Numerical Methods in Engineering 1992; 33(2):307-331, doi:10.1002/nme.1620330206. 
18. Lens E, Cardona A. An energy preserving/decaying scheme for nonlinearly constrained multibody systems. Multibody System Dynamics 2007; 18(3):435-470, doi:10.1007/s11044-007-9049-3.

19. Krenk S. State-space time integration with energy control and fourth-order accuracy for linear dynamic systems. International Journal for Numerical Methods in Engineering 2006; 65:595-619, doi:10.1002/nme.1449.

20. Wood WL. Numerical integration of structural dynamics equations including natural damping and periodic forcing terms. International Journal for Numerical Methods in Engineering 1981; $\mathbf{1 7}(2): 281-289$, doi:10.1002/nme.1620170211.

21. Fung TC. Unconditionally stable higher-order Newmark methods by sub-stepping procedure. Computer Methods in Applied Mechanics and Engineering 1997; 147:61-84, doi:10.1016/S00457825(96)01243-1.

22. Idesman AV. A new high-order accurate continuous Galerkin method for linear elastodynamics problems. Computational Mechanics 2007; 40:261-279, doi:10.1007/S00466-006-0096-Z.

23. Fung TC. Weighting parameters for unconditionally stable higher-order accurate time step integration algorithms. part 1-first-order equations. International Journal for Numerical Methods in Engineering 1999; 45(8):941-970, doi:10.1002/(SICI)1097-0207(19990720)45:8<941::AIDNME612>3.0.CO;2-S.

24. Bottasso CL, Borri M, Trainelli L. Integration of elastic multibody systems by invariant conserving/dissipating algorithms. II. Numerical schemes and applications. Computer Methods in Applied Mechanics and Engineering 2001; 190:3701-3733, doi:10.1016/S0045-7825(00)00285-1.

25. Bauchau OA, Bottasso CL, Trainelli L. Robust integration schemes for flexible multibody systems. Computer Methods in Applied Mechanics and Engineering 2003; 192:395-420, doi:10.1016/S00457825(02)00519-4.

26. Bottasso CL, Trainelli L. An Attempt at the Classification of Energy Decaying Schemes for Structural and Multibody Dynamics. Multibody System Dynamics 2004; 12:173-185, doi: 10.1023/B:MUBO.0000044418.23751.fe.

27. Hespanha J. Linear Systems Theory. Princeton University Press, 2009.

28. Bathe KJ, Noh G. Insight into an implicit time integration scheme for structural dynamics. Computers \& Structures 2012; 98-99(0):1-6, doi:10.1016/j.compstruc.2012.01.009.

29. Park KC, Lim SJ, Huh H. A method for computation of discontinuous wave propagation in heterogeneous solids: basic algorithm description and application to one-dimensional problems. International Journal for Numerical Methods in Engineering 2012; 91(6):622-643, doi: $10.1002 /$ nme.4285.

30. Hilber HM, Hughes TJR, Taylor RL. Improved numerical dissipation for time integration algorithms in structural dynamics. Earthquake Engineering $\&$ Structural Dynamics 1977; 5:283-292, doi: 10.1002/eqe.4290050306.

31. Wood WL, Bossak M, Zienkiewicz OC. An alpha modification of newmark's method. International Journal for Numerical Methods in Engineering 1980; 15(10):1562-1566, doi: 10.1002/nme.1620151011.

32. Li XD, Wiberg NE. Structural Dynamic Analysis by a Time-Discontinuous Galerkin Finite Element Method. International Journal for Numerical Methods in Engineering 1996; 39:2131-2152, doi: 10.1002/(SICI)1097-0207(19960630)39:12<2131::AID-NME947>3.0.CO;2-Z.

33. Cellier FE, Kofman E. Continuous System Simulation. Springer Science+Business Media, Inc., 2006.

34. Wood WL. A unified set of single step algorithms. Part 2: Theory. International Journal for Numerical Methods in Engineering 1984; 20(12):2303-2309, doi:10.1002/nme.1620201210.

35. Hulbert GM, Chung J. The unimportance of the spurious root of time integration algorithms for structural dynamics. Communications in Numerical Methods in Engineering 1994; 10:591-597, doi:10.1002/cnm.1640100803.

36. Romero I. On the stability and convergence of fully discrete solutions in linear elastodynamics. Computer Methods in Applied Mechanics and Engineering 2002; 191:3857-3882, doi: 10.1016/S0045-7825(02)00320-1.

37. Hughes TJR, Hulbert GM. Space-time finite element methods for elastodynamics: Formulations and error estimates. Computer Methods in Applied Mechanics and Engineering 1988; 66(3):339363, doi:10.1016/0045-7825(88)90006-0.

38. Bottasso CL. A new look at finite elements in time: a variational interpretation of Runge-Kutta methods. Applied Numerical Mathematics 1997; 25(4):355-368, doi:10.1016/S0168-9274(97)00072$\mathrm{X}$. 
39. Borri M, Bottasso CL, Trainelli L. An invariant-preserving approach to robust finite-element multibody simulation. Zeitschrift für Angewandte Mathematik und Mechanik 2003; 83:663-676, doi:10.1002/zamm.200310065.

40. Guttman L. Enlargement Methods for Computing the Inverse Matrix. The Annals of Mathematical Statistics 1946; 17:336-343, doi:10.1214/aoms/1177730946. 